\title{
Effects of genotype and environment on seed quality traits variability in interspecific cross-derived Brassica lines
}

\author{
Janetta Niemann (1) · Jan Bocianowski • Andrzej Wojciechowski
}

Received: 12 November 2017 / Accepted: 24 September 2018/Published online: 1 October 2018

(C) The Author(s) 2018

\begin{abstract}
Genotype (G), environment [i.e. year $(\mathrm{Y})$ and location $(\mathrm{L})]$ and their interaction (GYL) play an important role in the final expression of quality attributes. A multi-environment trial in selected interspecific cross-derived Brassica lines was conducted to evaluate the magnitude of G, Y, L and GYL effects on seed quality traits of Brassica genotypes under three locations in Poland, during the 2011-2013 cropping season. The oil, protein, fiber (Acid Detergent Fiber and Neutral Detergent Fiber) as well as glucosinolate contents was determined by near-infrared reflectance spectroscopy (NIRS) and significant differences were observed between tested Brassica genotypes and across harvesting years and growing locations. Generally, all tested hybrid lines displayed wider genetic variability for studied quality traits than control genotypes. Analysis of variance indicated that the main effects of genotypes, years and locations as well as all interactions were significant for all traits of study (except year $\times$ location interaction for glucosinolates). However, location had the most significant
\end{abstract}

J. Niemann $(\bowtie) \cdot$ A. Wojciechowski

Department of Genetics and Plant Breeding, Poznań

University of Life Sciences, Dojazd 11, 60-632 Poznań,

Poland

e-mail: niemann@up.poznan.pl

\section{J. Bocianowski}

Department of Mathematical and Statistical Methods, Poznań University of Life Sciences, Wojska Polskiego 28, 60-637 Poznań, Poland effect on oil, protein and fiber content while genotype had significant impact on glucosinolates content in Brassica seeds. Moreover, the individual lines having combination of desirable traits were also identified from $\mathrm{F}_{5}$ to $\mathrm{F}_{7}$ generations of tested hybrids.

Keywords Brassica lines - Seed quality traits · Genotype-environment interaction · Variability

\section{Introduction}

Brassica is the most economically important genus in the Brassicaceae family. Due to substantial progress in breeding and cultivation practice, two major members of the Brassica genus commonly known as rapeseed (Brassica napus L.) and mustards have become one of the worldwide most principal source of vegetable oil (Rani et al. 2013). Especially in several European countries with cool-temperate climates oilseed rape (B. napus) with 'double-low' seed quality (Canola) dominates field crop production. Poland and Great Britain have been ranked interchangeably in sixth place in the production of winter oilseed rape (Brassica napus L.) in the world, after China, Canada, India, Germany and France (Ratajczak et al. 2017). In Poland, winter oilseed rape is the most important oil-protein plant. It provides edible vegetable oil for human consumption and proteins for the 
feed industry, and has recently gained increasing interest as a source of biodiesel (Huang et al. 2016). Traditional breeding, as well as, modern biotechnological tools has led to the improvement of various quantitative and qualitative traits in Brassica. At the same time, modern crop breeding has resulted in the reduction of genetic variability in most of the crop species (Tanksley and Nelson 1996). From our previous work it was found that one of the most effective methods for increasing the variability of traits that impacts the quality of rapeseed products is interspecific hybridization (Niemann et al. 2012). In order to combine desirable genes found in two or more different varieties, planned hybridization between carefully selected parents is practiced within or among species. The plant breeder usually keeps such ideal plants in mind that combines a majority of desirable characteristics. These may be general and/or specific traits that could contribute to the improved adaptation of plants to the environment and could enhance yield with better quality seed. Generally, in Brassica napus breeding, traits related to commercial success are of highest importance (Friedt and Snowdon 2010). For this reason rapeseed breeding strategies are oriented on developnig new cultivars with high oil, protein as well as low erucic acid, glucosinolate and fiber content (Möllers 2004; Abbadi and Leckband 2011; Zou et al. 2016; Körber et al. 2016). For this purpose knowledge of genetic diversity is indispensible in the development of commercial cultivars (Ahmad et al. 2013; Liu et al. 2016).

The content of oil, protein and anti-nutritive substances like glucosinolates and fiber in rapeseeds decide on their utility value (Bell 1984; Vageeshbabu and Chopra 1997; Liu et al. 2012). Brassica napus is planted for oil production and, therefore, an maximization of oil content in the seeds is a major goal in the breeding process (Zhao et al. 2007; Würschum et al. 2012). In general, the oil content in the Brassica seeds varies from 30 to $45 \%$ depending on the species, the variety and climatic conditions and is mainly driven by the composition of fatty acid components of erucic acid (C22:1), stearic acid (C18:0), oleic acid (C18:1), linoleic acid (C18:2), and linolenic acid (C18:3) (Velasco and Becker 1998; Möllers 2004). In addition, the oil content of Brassica oilseed meal contains about $40 \%$ protein with a well-balanced amino acid composition (Khan et al. 2008), but lower than would be desired. Moreover, plant breeders have strongly reduced the levels of the unhealthy and uneatable glucosinolates in the seeds $(<30 \mathrm{uM} / \mathrm{g})$ to be able to use the protein-rich seed cake as an animal feed supplement (Halkier and Gershenzon 2006). Besides, the presence of high fibre fraction in oilseed rape meal, which is mostly located in seed hull, is the major obstacle for its use in animal feeding. The fibre fraction is poorly digestible and essentially dilutes the available energy and protein. Consequently, oilseed rape meal has less metabolizable energy and reduces the value of the meal relatively to soybean meal. Fibre contents are related to plant cell walls components, namely cellulose, hemicellulose and lignin. The proportion of hemicellulose, cellulose and lignin can be quantified as neutral detergent fibre (NDF), acid detergent fibre (ADF) and acid detergent lignin (ADL). The NDF fraction consists (predominantly) of hemicellulose, cellulose and lignin content, while the ADF fraction comprises cellulose and lignin. ADL represents the non-digestible lignin fraction. To improve the quality of oilseed rape meal, breeding research has been undertaken to select genetic material with lower fibre content.

It is known that all of these seed quality traits are influenced by genetic and environmental factors, as well as agricultural technology (Feng et al. 2012; Ratajczak et al. 2017), so their precise estimation requires phenotyping in replicated multi-environmental field trials. It is important to determine and quantify the extent to which factors like the environment and genotype $x$ environment interaction contribute to variations in each Brassica quality parameter.

The objectives of this research were (1) to determine the relative contributions of G, Y, L and GYL to the variation in seed quality traits of twenty-five Brassica genotypes tested across three locations, (2) to analyze which factor has the most significant effect on tested traits, and (3) to find the best Brassica genotypes based on quality traits.

\section{Materials and methods}

Plant material for field trials consisted of 25 winter rapeseed genotypes i.e.: B. napus cv. Californium (open pollinated variety), twenty-three interspecific cross-derived Brassica lines and male sterile line of $\mathrm{F}_{8}$ generation of B. napus (MS8). MS8 line was selected from resynthesized oilseed rape (B. rapa ssp. 
chinensis $\times B$. oleracea var. gemmifera) using in vitro cultures of isolated embryos in Department of Genetics and Plant Breeding, Poznan University of Life Sciences (PULS) (Table 1). Seeds of B. napus cv. Californium and MS8 line originated from the resources of the Department of Genetics and Plant Breeding of PULS, while seeds of paternal genotypes i.e. Brassica rapa ssp. pekinensis, Brassica rapa ssp. trilocularis, Brassica carinata and Brassica juncea came from the Warwick HRI Genetic Resources Unit. In order to obtain interspecific cross-derived Brassica lines, interspecific hybridizations were carried out in the glasshouse and performed with the application of in vitro culture of isolated embryos according to the method described by Niemann et al. (2012). All interspecific cross-derived lines were sister-pollinated (five plants were enclosed in one paper bag during flowering) for four generations in order to stabilize the fertility. $\mathrm{F}_{5}-\mathrm{F}_{7}$ generation of tested lines and control genotypes were selected from the rapeseed breeding program of Department of the Genetics and Plant Breeding of PULS. During seasons 2010/2011, 2011/2012 and 2012/2013 twenty-five Brassica genotypes were grown in three different locations i.e. in the PULS experimental station Dłoń located $100 \mathrm{~km}$ south of Poznań and in Złotniki located $15 \mathrm{~km}$ west from Poznań as well as in the experimental fields in Poznań-Sołacz. These locations have different types of soil and weather conditions (Tables 2,3). The field trials at all locations were arranged in a randomized complete block design with three replicates. Each genotype was grown in a 3 row plot of $9.0 \mathrm{~m}^{2}$ with a 0.30 row distance and a sawing density of 60 seeds $/ \mathrm{m}^{2}$.

Quality analysis

The seed samples for the analysis of fiber, protein, oil and glucosinolates content were collected from five
Table 1 Code and origin of the genotypes tested

\begin{tabular}{|c|c|}
\hline Code & Genotype \\
\hline 1 & B. napus cv. Californium \\
\hline 2 & B. napus line MS $8 \times B$. rapa ssp. pekinensis \\
\hline 3 & B. napus line MS8 $\times$ B. rapa ssp. pekinensis \\
\hline 4 & B. napus line MS8 $\times$ B. rapa ssp. pekinensis \\
\hline 5 & B. napus line MS $8 \times B$. carinata \\
\hline 6 & B. napus line MS8 $\times$ B. rapa ssp. pekinensis \\
\hline 7 & B. napus line MS $8 \times B$. carinata \\
\hline 8 & B. napus line MS $8 \times B$. carinata \\
\hline 9 & B. napus line MS8 $\times$ B. carinata \\
\hline 10 & B. napus line MS $8 \times B$. rapa ssp. trilocularis $c v$. Yellow Sarson \\
\hline 11 & B. napus line MS $8 \times B$. rapa ssp. trilocularis $c v$. Yellow Sarson \\
\hline 12 & B. napus line MS $8 \times B$. rapa ssp. trilocularis $c v$. Yellow Sarson \\
\hline 13 & B. napus line MS8 $\times$ B. rapa ssp. pekinensis \\
\hline 14 & B. napus line MS8 $\times$ B. rapa ssp. pekinensis \\
\hline 15 & B. napus line MS8 $\times$ B. rapa ssp. pekinensis \\
\hline 16 & B. napus line MS8 $\times$ B. rapa ssp. pekinensis \\
\hline 17 & B. napus line MS8 $\times$ B. rapa ssp. pekinensis \\
\hline 18 & B. napus line MS $8 \times B$. rapa ssp. pekinensis \\
\hline 19 & B. napus line MS $8 \times B$. juncea \\
\hline 20 & B. napus line MS8 $\times$ B. rapa ssp. trilocularis $c v$. Yellow Sarson \\
\hline 21 & B. napus line MS $8 \times B$. rapa ssp. trilocularis $c v$. Yellow Sarson \\
\hline 22 & B. napus line MS8 $\times$ B. rapa ssp. pekinensis \\
\hline 23 & B. napus line MS $8 \times B$. carinata \\
\hline 24 & B. napus line MS $8 \times B$. juncea \\
\hline 25 & B. napus line MS8 \\
\hline
\end{tabular}


Table 2 Environments used in the study and their main characteristics

\begin{tabular}{llllll}
\hline Environment & Longitude $(\mathrm{E})$ & Latitude $(\mathrm{N})$ & Soil quality class & Soil type & Complex of agricultural usefulness \\
\hline Dłoń & $17^{\circ} 04^{\prime} 10^{\prime \prime}$ & $51^{\circ} 41^{\prime} 23^{\prime \prime}$ & III & Heavy & Good rye \\
Poznań-Sołacz & $16^{\circ} 54^{\prime} 26^{\prime \prime}$ & $52^{\circ} 25^{\prime} 18^{\prime \prime}$ & IV & Light, sandy & Good wheat \\
Złotniki & $16^{\circ} 50^{\prime} 41^{\prime \prime}$ & $52^{\circ} 29^{\prime} 35^{\prime \prime}$ & IVa & Luvisols & Very good rye \\
\hline
\end{tabular}

Table 3 Meteorological conditions in Dłoń, Poznań-Sołacz and Złotniki during the vegetation season of winter oilseed rape in 2011, 2012 and 2013

\begin{tabular}{|c|c|c|c|c|c|c|c|c|c|}
\hline \multirow[t]{2}{*}{ Basic weather parameters } & \multicolumn{3}{|l|}{ Dłoń } & \multicolumn{3}{|c|}{ Poznań-Sołacz } & \multicolumn{3}{|c|}{ Złotniki } \\
\hline & 2011 & 2012 & 2013 & 2011 & 2012 & 2013 & 2011 & 2012 & 2013 \\
\hline Mean annual temperature $\left({ }^{\circ} \mathrm{C}\right)$ & 10 & 10.1 & 9.7 & 9.6 & 9.4 & 9.2 & 15.2 & 9.1 & 9.8 \\
\hline Sum of precipitation $(\mathrm{mm})$ in whole year & 465 & 631 & 565 & 477 & 514 & 607 & 402 & 678 & 581 \\
\hline
\end{tabular}

self-pollinated plants from each tested line. The fiber content in the Brassica lines and control seeds was expressed as the average value of the neutral detergent fiber (NDF) and the acid detergent fiber (ADF) as a subfraction of the NDF. The seed quality traits content was determined by near-infrared reflectance spectroscopy (NIRS) (Velasco et al. 1999). These analyses were performed at the Małyszyn Laboratory that belongs to Plant Breeding Strzelce in triplicate. For NIRS analysis minimum $3 \mathrm{~g}$ of intact seeds were used. Every seed probe was scanned on the NIRS Systems monochromator model 6500 (NIR Systems, Inc. Silver Springers, MD, USA).

\section{Statistical analysis}

Firstly, the normality of the distributions of the studied traits (neutral detergent fiber-NDF, acid detergent fiber-ADF, as a subfraction of the NDF, protein, oil content and glucosinolates) were tested using Shapiro-Wilk's normality test (Shapiro and Wilk 1965). Multivariate analysis of variance (MANOVA) was performed on the basis of following model using a procedure MANOVA in GenStat 17th edition: $\mathbf{Y}=\mathbf{X T}+\mathbf{E}$, where: $\mathbf{Y}$ is $(n \times p)$-dimensional matrix of observations, $n$ is number of all observations, $p$ is number of traits $(p=5), \mathbf{X}$ is $(n \times k)$-dimensional matrix of design, $k$ is number of genotypes $(k=25), \mathbf{T}$ is $(k \times p)$-dimensional matrix of unknown effects, $\mathbf{E}$-is $(n \times p)$-dimensional matrix of residuals. Nextly, three-way analysis of variance (ANOVA) was carried out to determine the effects of genotypes, years and locations and the interactions: genotypes $\times$ years, genotypes $\times$ locations, years $\times$ locations and genotypes $\times$ years $\times$ locations - on the variability of studied traits. The minimal and maximal values of traits as well as arithmetical means and standard deviations were calculated. Moreover, the Fisher's least significant differences (LSDs) were also estimated at the significance level $\alpha=0.05$. The relationships between observed traits were assessed on the basis of Pearson's correlation coefficients using FCORRELATION procedure in GenStat 17th edition for each environment independent. Results were also analysed using multivariate methods. The canonical variate analysis was applied in order to present multitrait assessment of similarity of tested genotypes in a lower number of dimensions with the least possible loss of information (Rencher 1992). This makes it possible to illustrate variation in genotypes in terms of all observed traits in the graphic form. Mahalanobis' distance was suggested as a measure of "polytrait" genotypes similarity (Seidler-Łożykowska and Bocianowski 2012), whose significance was verified by means of critical value $D_{\alpha}$ called "the least significant distance" (Mahalanobis 1936). Mahalanobis' distances were calculated for 
genotypes in each environment independent as well as for all environments data jointly. All the analyses were conducted using the GenStat v. 17 statistical software package (Payne et al. 2012).

\section{Results}

All studied traits have a normal distribution as well as a multivariate normality. Results of MANOVA indicate that the genotypes (Wilk's $\lambda=0.0448$; $\mathrm{F}_{24,3148}=113.51 ; P<0.0001$ ), years (Wilk's $\lambda=$ $\left.0.7618 ; \mathrm{F}_{2,3148}=91.63 ; P<0.0001\right)$ and locations (Wilk's $\lambda=0.1305 ; \quad \mathrm{F}_{2,3148}=1112 ; \quad P<0.0001$ ) were significant different for all five traits. Moreover, interactions: genotype $\times$ years (Wilk's $\lambda=0.7047$; $\left.\mathrm{F}_{48,3148}=4.75 ; \quad P<0.0001\right)$, genotype $\times$ location (Wilk's $\lambda=0.1480 ; \mathrm{F}_{48,3148}=30.52 ; P<0.0001$ ), years $\times$ locations (Wilk's $\lambda=0.6796 ; \quad F_{4,3148}=$ 64.39; $P<0.0001)$ and genotype $\times$ years $\times$ locations (Wilk's $\lambda=0.2344 ; \quad \mathrm{F}_{96,3148}=11.03 ; \quad P<0.0001$ ) were significant. Analysis of variance indicated that the main effects of genotypes, years and locations as well as all interactions were significat for all the traits of study (except year $\times$ location interaction for glucosinolates) (Table 4). However, the obtained data showed that the most significant effect on oil, protein and fiber content in the Brassica seeds had environment i.e. location, while genotype had the biggest impact on glucosinolates content.
Minimal, maximal and mean values as well as standard deviations for NDF, ADF, protein, oil and glucosinolates content were presented in Tables 5, 6, 7, 8 and 9, respectively.

Generally, all tested cross-derived Brassica lines displayed a great increase in the range of variability for the studied quality traits when compared to the control genotypes. Moreover, the highest mean values of fiber, oil and glucosinolates were observed in Dłon, although protein content in tested lines in total was slightly higher in Złotniki, where mainly the lowest values of all studied traits were noticed (Tables 5, 6, 7, $8,9)$. Both, the highest and the lowest NDF mean values were observed in lines harvested in Dłoń (29.89 and $18.61 \%$ respectively), however the differences occured between tested genotypes i.e. in MS8 $\times B$. rapa ssp. trilocularis and $\mathrm{MS} 8 \times B$. rapa $\mathrm{ssp}$. pekinensis adequately (Table 5). Similarly, the highest ADF mean values were observed in the lines harvested in Dłon from MS8 $\times$ B. rapa ssp. pekinensis seeds $(26.68 \%)$ but the lowest content was noticed in Złotniki from MS8 $\times$ B. juncea-17.1\% (Table 6). The highest protein mean value was observed in Złotniki (23.80\%), while the lowest in Poznań-Sołacz $(16.65 \%)$ in MS8 $\times$ B. rapa ssp. pekinensis lines in 2012 (lines number 3 and 11, respectively) (Table 7). The highest oil mean value was observed in Dłoń in line number $16(46.96 \%)$, ranged from 44.52 to $49.89 \%$, but the minimum value was noticed in Złotniki $(35.35 \%)$ in line number 17 (Table 8 ). The minimal glucosinolates content was observed in

Table 4 Mean squares from three-way analysis of variance for five observed traits

\begin{tabular}{|c|c|c|c|c|c|c|}
\hline Source of variation & d.f. & NDF & $\mathrm{ADF}$ & Protein content & Oil content & Glucosinolate content \\
\hline Repl & 2 & 3.99 & 21.45 & 6.81 & 0.87 & 0.4 \\
\hline Genotyp (G) & 24 & $345.30 * * *$ & $70.60 * * *$ & $65.63 * * *$ & $108.25 * * *$ & $18,469.81 * * *$ \\
\hline Year $(Y)$ & 2 & $551.38 * * *$ & $70.81 * * *$ & $154.85 * * *$ & $85.55 * * *$ & $137.83 *$ \\
\hline Location (L) & 2 & $1552.08 * * *$ & $2540.92 * * *$ & $3584.18 * * *$ & $2088.25 * * *$ & $2874.3 * * *$ \\
\hline $\mathrm{G} \times \mathrm{Y}$ & 48 & $13.86 * * *$ & $3.87 * * *$ & $2.80 * * *$ & $9.34 * * *$ & $54.89 * *$ \\
\hline $\mathrm{G} \times \mathrm{L}$ & 48 & $62.74 * * *$ & $37.30 * * *$ & $46.91 * * *$ & $88.27 * * *$ & $288.89 * * *$ \\
\hline $\mathrm{Y} \times \mathrm{L}$ & 4 & $370.33 * * *$ & $66.86^{* * *}$ & $96.03 * * *$ & $27.54 * * *$ & $22.68^{\mathrm{ns}}$ \\
\hline $\mathrm{G} \times \mathrm{Y} \times \mathrm{L}$ & 96 & $17.05 * * *$ & $19.78 * * *$ & $12.84 * * *$ & $22.69 * * *$ & $53.07 * * *$ \\
\hline Residual & 3148 & 1.75 & 0.63 & 0.84 & 4.06 & 31.76 \\
\hline
\end{tabular}

$n s$ Not significant

$* P<0.05 ; * * P<0.01 ; * * * P<0.001$ 
Table 5 Neutral detergent fiber content (\%) in twenty-five Brassica genotypes tested in nine environments

\begin{tabular}{|c|c|c|c|c|c|c|c|c|c|c|c|c|c|}
\hline \multirow{2}{*}{$\begin{array}{l}\text { Location } \\
\text { Genotyp }\end{array}$} & \multirow[t]{2}{*}{ Year } & \multicolumn{4}{|l|}{ Dlon } & \multicolumn{4}{|c|}{ Poznań-Sołacz } & \multicolumn{4}{|c|}{ Złotniki } \\
\hline & & Mean & Min & Max & SD & Mean & Min & Max & SD & Mean & Min & Max & SD \\
\hline \multirow[t]{3}{*}{1} & 2011 & 28.82 & 28.53 & 29.21 & 0.17 & 27.7 & 26.25 & 30.21 & 1.03 & 26.87 & 26.09 & 28.45 & 0.53 \\
\hline & 2012 & 27.87 & 25.54 & 29.33 & 1.39 & 27.61 & 25.55 & 33.87 & 2.16 & 26.42 & 25.67 & 28.45 & 0.74 \\
\hline & 2013 & 28.89 & 27.44 & 30.23 & 0.95 & 29.14 & 26.94 & 31.95 & 1.52 & 26.22 & 25.45 & 28.41 & 0.85 \\
\hline \multirow[t]{3}{*}{2} & 2011 & 22.66 & 18.9 & 23.99 & 1.50 & 26.54 & 26.23 & 27.07 & 0.22 & 23.31 & 22.95 & 23.79 & 0.22 \\
\hline & 2012 & 23.22 & 22.8 & 23.79 & 0.27 & 26.72 & 26.14 & 27.75 & 0.52 & 24.74 & 21.25 & 27.23 & 1.65 \\
\hline & 2013 & 25.49 & 22.26 & 29.2 & 2.09 & 26.81 & 25.16 & 28.68 & 1.02 & 23.19 & 22.16 & 25.56 & 0.85 \\
\hline \multirow[t]{3}{*}{3} & 2011 & 23.43 & 22.23 & 25.56 & 0.74 & 27.19 & 26.73 & 27.62 & 0.28 & 22.77 & 19.61 & 25.84 & 2.18 \\
\hline & 2012 & 24.74 & 21.25 & 27.23 & 1.65 & 27.18 & 26.55 & 27.69 & 0.33 & 22.78 & 21.79 & 25.56 & 1.06 \\
\hline & 2013 & 22.09 & 20.55 & 25.39 & 1.12 & 27.14 & 25.61 & 28.82 & 1.10 & 26.36 & 23.06 & 28.96 & 2.00 \\
\hline \multirow[t]{3}{*}{4} & 2011 & 24.37 & 20.58 & 27.76 & 2.86 & 28.17 & 27.66 & 29.03 & 0.29 & 26.83 & 26.38 & 28.27 & 0.45 \\
\hline & 2012 & 21.21 & 20.13 & 28.23 & 1.97 & 28.11 & 27.51 & 29.03 & 0.35 & 26.67 & 26.15 & 28.27 & 0.53 \\
\hline & 2013 & 28.76 & 25.99 & 31.31 & 1.47 & 28.15 & 25.71 & 30.75 & 1.24 & 26.94 & 25.84 & 28.74 & 1.02 \\
\hline \multirow[t]{3}{*}{5} & 2011 & 20.96 & 20.49 & 21.36 & 0.24 & 26.11 & 23.76 & 27.79 & 0.97 & 22.08 & 21.3 & 23.55 & 0.59 \\
\hline & 2012 & 21.9 & 21.15 & 23.55 & 0.65 & 26.15 & 23.33 & 28.56 & 1.48 & 22.28 & 20.38 & 26.95 & 2.18 \\
\hline & 2013 & 23.39 & 21.23 & 26.97 & 2.17 & 26.98 & 23.38 & 28.43 & 1.38 & 22.15 & 20.77 & 23.8 & 1.02 \\
\hline \multirow[t]{3}{*}{6} & 2011 & 21.18 & 19 & 23.96 & 2.31 & 20.9 & 20.23 & 21.61 & 0.42 & 23.7 & 23.04 & 25.77 & 0.64 \\
\hline & 2012 & 20.68 & 19.19 & 23.95 & 1.37 & 21.07 & 20.08 & 23.98 & 1.15 & 23.67 & 23 & 25.77 & 0.65 \\
\hline & 2013 & 21.87 & 19.23 & 23.92 & 1.63 & 23.66 & 20.05 & 27 & 2.35 & 23.47 & 21.13 & 25.52 & 1.05 \\
\hline \multirow[t]{3}{*}{7} & 2011 & 27.7 & 20.36 & 28.92 & 2.08 & 23.02 & 22.51 & 23.45 & 0.28 & 23.71 & 23.18 & 25.67 & 0.58 \\
\hline & 2012 & 20.37 & 19.16 & 27.09 & 2.08 & 22.87 & 22.14 & 23.8 & 0.45 & 24.16 & 23.13 & 25.67 & 0.85 \\
\hline & 2013 & 27.98 & 25.26 & 30.48 & 1.42 & 23.31 & 21.33 & 29.05 & 1.74 & 23.94 & 22.16 & 25.88 & 1.13 \\
\hline \multirow[t]{3}{*}{8} & 2011 & 29 & 27.21 & 29.95 & 0.59 & 28.45 & 28 & 29.18 & 0.33 & 23.91 & 23.19 & 25.95 & 0.71 \\
\hline & 2012 & 27.73 & 23.51 & 29.95 & 1.92 & 28.19 & 27.17 & 29.18 & 0.52 & 23.88 & 23.01 & 25.95 & 0.72 \\
\hline & 2013 & 29.08 & 26.48 & 31 & 1.19 & 28.44 & 26.71 & 30.18 & 0.85 & 23.84 & 23.12 & 25.92 & 0.87 \\
\hline \multirow[t]{3}{*}{9} & 2011 & 26.21 & 23.13 & 26.87 & 1.23 & 26.78 & 26 & 28.45 & 0.64 & 27.24 & 26.93 & 27.54 & 0.19 \\
\hline & 2012 & 26.55 & 23.58 & 29.6 & 1.71 & 26.71 & 25.84 & 28.45 & 0.65 & 26.25 & 23.13 & 26.91 & 1.25 \\
\hline & 2013 & 25.08 & 22.46 & 26.98 & 1.76 & 26.72 & 23.83 & 28.82 & 1.47 & 27.42 & 25.56 & 29.24 & 1.13 \\
\hline \multirow[t]{3}{*}{10} & 2011 & 29.8 & 29.63 & 30.55 & 0.22 & 28.69 & 28.29 & 29.04 & 0.23 & 26.64 & 26.44 & 26.96 & 0.14 \\
\hline & 2012 & 26.97 & 21.22 & 30.55 & 4.10 & 28.85 & 28.29 & 29.38 & 0.32 & 26.61 & 26.25 & 26.96 & 0.18 \\
\hline & 2013 & 29.61 & 27 & 31.63 & 1.27 & 27.87 & 23.42 & 29.66 & 1.97 & 26.65 & 25.79 & 27.36 & 0.49 \\
\hline \multirow[t]{3}{*}{11} & 2011 & 29.43 & 28.9 & 30.44 & 0.42 & 29.89 & 29.04 & 30.5 & 0.35 & 26.64 & 25.3 & 29.8 & 1.83 \\
\hline & 2012 & 24.21 & 20.92 & 30.14 & 3.70 & 29.9 & 28.2 & 30.87 & 0.63 & 26.62 & 25.2 & 29.61 & 1.82 \\
\hline & 2013 & 29.54 & 27.82 & 31.6 & 1.02 & 29.81 & 28.45 & 31.61 & 0.78 & 27.04 & 25.24 & 29.88 & 1.61 \\
\hline \multirow[t]{3}{*}{12} & 2011 & 29.09 & 28.86 & 29.23 & 0.10 & 28.41 & 27.78 & 28.64 & 0.23 & 27.84 & 27.5 & 28.55 & 0.27 \\
\hline & 2012 & 27.76 & 27.23 & 28.55 & 0.33 & 28.27 & 27.65 & 28.9 & 0.38 & 25.9 & 20.07 & 29.3 & 4.15 \\
\hline & 2013 & 29.17 & 27.08 & 30.33 & 0.83 & 28.66 & 27.58 & 30.21 & 0.97 & 27.72 & 23.89 & 28.99 & 1.18 \\
\hline \multirow[t]{3}{*}{13} & 2011 & 27.81 & 27.43 & 28.11 & 0.20 & 26.63 & 25.87 & 27.8 & 0.51 & 24.92 & 23.17 & 28.77 & 2.06 \\
\hline & 2012 & 26.43 & 22.77 & 27.95 & 2.02 & 26.64 & 25.86 & 27.8 & 0.49 & 24.68 & 23.09 & 27.45 & 1.73 \\
\hline & 2013 & 27.8 & 26.01 & 29.56 & 1.07 & 26.81 & 25.01 & 28.33 & 1.08 & 24.63 & 23.02 & 27.51 & 1.45 \\
\hline \multirow[t]{3}{*}{14} & 2011 & 25.63 & 18.35 & 26.54 & 2.04 & 26.52 & 25.2 & 27.8 & 0.62 & 23.64 & 23.07 & 23.85 & 0.18 \\
\hline & 2012 & 18.61 & 15.31 & 26.71 & 2.46 & 25.25 & 23.31 & 26.57 & 0.65 & 23.49 & 23 & 23.84 & 0.30 \\
\hline & 2013 & 23.64 & 23.02 & 27.5 & 1.12 & 25.11 & 23.4 & 26.94 & 1.08 & 25.47 & 23.19 & 27.34 & 1.38 \\
\hline 15 & 2011 & 26.49 & 20.1 & 27.36 & 1.78 & 26.72 & 25.89 & 27.01 & 0.25 & 23.61 & 23.08 & 23.93 & 0.21 \\
\hline
\end{tabular}


Table 5 continued

\begin{tabular}{|c|c|c|c|c|c|c|c|c|c|c|c|c|c|}
\hline \multirow{2}{*}{$\begin{array}{l}\text { Location } \\
\text { Genotyp }\end{array}$} & \multirow[t]{2}{*}{ Year } & \multicolumn{4}{|l|}{ Dlon } & \multicolumn{4}{|c|}{ Poznań-Sołacz } & \multicolumn{4}{|c|}{ Złotniki } \\
\hline & & Mean & Min & $\operatorname{Max}$ & SD & Mean & Min & $\operatorname{Max}$ & SD & Mean & Min & $\operatorname{Max}$ & SD \\
\hline & 2012 & 20.46 & 19.04 & 27.41 & 1.99 & 26.63 & 25.89 & 26.85 & 0.25 & 23.6 & 23.08 & 25 & 0.46 \\
\hline & 2013 & 27.01 & 25.52 & 28.24 & 0.83 & 26.74 & 25.39 & 28.32 & 0.74 & 23.16 & 22.04 & 25.49 & 0.83 \\
\hline \multirow[t]{3}{*}{16} & 2011 & 25.22 & 21.02 & 25.85 & 1.18 & 26.53 & 25.53 & 27.62 & 0.59 & 22.33 & 21.69 & 22.62 & 0.24 \\
\hline & 2012 & 21.24 & 20.35 & 25.31 & 1.21 & 25.55 & 23.75 & 26.82 & 1.20 & 22.12 & 21.51 & 22.58 & 0.36 \\
\hline & 2013 & 25.71 & 23.04 & 28.6 & 1.79 & 26.03 & 23.67 & 27.91 & 1.30 & 22.17 & 21.15 & 23.65 & 0.87 \\
\hline \multirow[t]{3}{*}{17} & 2011 & 26.29 & 21.3 & 26.93 & 1.42 & 25.61 & 25.39 & 25.81 & 0.12 & 22.29 & 21.52 & 23.23 & 0.50 \\
\hline & 2012 & 20.42 & 19.15 & 22.17 & 0.99 & 25.54 & 25.11 & 26.17 & 0.31 & 22.03 & 21.11 & 23.23 & 0.67 \\
\hline & 2013 & 26.67 & 23.18 & 29.07 & 1.67 & 25.45 & 23.12 & 27.62 & 1.20 & 22.3 & 20.5 & 23.31 & 0.83 \\
\hline \multirow[t]{3}{*}{18} & 2011 & 23.51 & 23.01 & 23.98 & 0.40 & 23.64 & 23.16 & 26.89 & 0.93 & 22.88 & 22.07 & 23.67 & 0.50 \\
\hline & 2012 & 21.88 & 18.67 & 23.72 & 2.23 & 23.63 & 23.13 & 26.89 & 0.94 & 22.79 & 21.63 & 23.8 & 0.64 \\
\hline & 2013 & 24.18 & 22.69 & 27.17 & 1.50 & 23.99 & 22.18 & 28.9 & 1.66 & 22.68 & 21.54 & 23.54 & 0.68 \\
\hline \multirow[t]{3}{*}{19} & 2011 & 26.22 & 23.94 & 27.1 & 0.68 & 26.46 & 25.95 & 26.8 & 0.21 & 23.81 & 22.02 & 27.45 & 1.74 \\
\hline & 2012 & 23.98 & 19.58 & 27.32 & 3.22 & 26.41 & 25.77 & 26.96 & 0.32 & 23.53 & 22.26 & 26.45 & 1.40 \\
\hline & 2013 & 25.74 & 23.05 & 30.23 & 2.42 & 25.65 & 23.46 & 27.65 & 1.42 & 23.19 & 21.31 & 25.92 & 1.19 \\
\hline \multirow[t]{3}{*}{20} & 2011 & 26.89 & 26.26 & 27.18 & 0.29 & 26.58 & 26.23 & 26.95 & 0.19 & 23.4 & 23.11 & 23.79 & 0.23 \\
\hline & 2012 & 23.9 & 19.11 & 27.43 & 3.67 & 26.46 & 25.84 & 26.8 & 0.29 & 23.32 & 23 & 23.79 & 0.26 \\
\hline & 2013 & 26.93 & 23.12 & 29.14 & 1.81 & 26.46 & 25.13 & 27.88 & 0.76 & 23.6 & 22.63 & 25.1 & 0.68 \\
\hline \multirow[t]{3}{*}{21} & 2011 & 27.63 & 26.18 & 29.77 & 0.79 & 26.51 & 26.18 & 26.96 & 0.22 & 22.19 & 21.48 & 22.55 & 0.26 \\
\hline & 2012 & 25.03 & 19.32 & 29.77 & 4.10 & 26.4 & 25.89 & 26.96 & 0.29 & 22.02 & 21.48 & 22.55 & 0.29 \\
\hline & 2013 & 27.8 & 23.2 & 30.36 & 1.76 & 26.5 & 25.16 & 27.8 & 0.79 & 22.18 & 20.78 & 23.29 & 0.70 \\
\hline \multirow[t]{3}{*}{22} & 2011 & 26.79 & 19.23 & 28.52 & 2.13 & 26.2 & 25.37 & 26.68 & 0.42 & 22.91 & 22.08 & 24.99 & 0.90 \\
\hline & 2012 & 20.66 & 18.77 & 26.29 & 2.22 & 25.53 & 23.26 & 26.68 & 1.29 & 23.18 & 22.16 & 24.97 & 1.11 \\
\hline & 2013 & 27.44 & 25.86 & 29.14 & 1.16 & 25.73 & 22.59 & 28.08 & 1.55 & 23.11 & 22.01 & 24.68 & 0.87 \\
\hline \multirow[t]{3}{*}{23} & 2011 & 26.12 & 21.43 & 26.93 & 1.33 & 25.33 & 23.43 & 25.97 & 0.74 & 23.29 & 22.75 & 23.5 & 0.23 \\
\hline & 2012 & 22.14 & 20.65 & 27.92 & 2.10 & 24.83 & 23.6 & 25.97 & 0.92 & 22.99 & 22.04 & 23.5 & 0.53 \\
\hline & 2013 & 26.24 & 23.6 & 28.76 & 1.57 & 25.45 & 23.24 & 27.52 & 1.52 & 22.99 & 21.87 & 23.72 & 0.62 \\
\hline \multirow[t]{3}{*}{24} & 2011 & 26.5 & 19.9 & 27.44 & 1.85 & 26.11 & 25.43 & 27.18 & 0.46 & 21.98 & 21.27 & 23.47 & 0.58 \\
\hline & 2012 & 20.45 & 19.19 & 26.65 & 1.96 & 25.72 & 23.52 & 27.18 & 0.97 & 21.77 & 20.99 & 23.47 & 0.71 \\
\hline & 2013 & 26.65 & 23.07 & 29.67 & 1.91 & 25.82 & 23.35 & 28.16 & 1.33 & 22.05 & 20.48 & 24 & 1.18 \\
\hline \multirow[t]{3}{*}{25} & 2011 & 27.53 & 22.03 & 28.43 & 1.55 & 27.05 & 26.11 & 27.94 & 0.59 & 22.54 & 22.1 & 23.51 & 0.40 \\
\hline & 2012 & 21.96 & 21.12 & 27.56 & 1.59 & 26.59 & 25.46 & 27.71 & 0.59 & 22.86 & 22.08 & 23.69 & 0.62 \\
\hline & 2013 & 27.76 & 25.42 & 30.17 & 1.48 & 26.31 & 23.98 & 27.98 & 1.06 & 23.14 & 20.72 & 23.88 & 0.88 \\
\hline $\mathrm{LSD}_{0.05}$ & \multicolumn{13}{|c|}{ G: 0.32, Y: 0.11, L: 0.11, GY: 0.55, GL: 0.55, YL: 0.19, GYL: 0.95} \\
\hline
\end{tabular}

Złotniki in MS8 $\times$ B. rapa lines and ranged from 6.1 to $69.37 \mathrm{umol} / \mathrm{g}$ seeds (Table 9).

NDF was positive correlated with ADF in all nine environments, except in Dłoń 2012 (Table 10). Protein content was negative correlated with NDF (except Dłoń 2012 and Złotniki 2012) and ADF (except Dłon 2012). Oil content was positive correlated with NDF and ADF in six environments; and negative correlated with protein content in eight environment (only in Złotniki 2012 these two traits were not correlated). Glucosinolates content and NDF were negative correlated in all nine environments. 
Table 6 Acid detergent fiber content (\%) in twenty-five Brassica genotypes tested in nine environments

\begin{tabular}{|c|c|c|c|c|c|c|c|c|c|c|c|c|c|}
\hline \multirow{2}{*}{$\begin{array}{l}\text { Location } \\
\text { Genotyp }\end{array}$} & \multirow[t]{2}{*}{ Year } & \multicolumn{4}{|l|}{ Dłon } & \multicolumn{4}{|c|}{ Poznań-Sołacz } & \multicolumn{4}{|c|}{ Złotniki } \\
\hline & & Mean & Min & Max & SD & Mean & Min & Max & SD & Mean & Min & Max & SD \\
\hline \multirow[t]{3}{*}{1} & 2011 & 22.51 & 22.3 & 22.59 & 0.09 & 21.94 & 20.57 & 23.64 & 0.82 & 20.59 & 19.89 & 22.03 & 0.47 \\
\hline & 2012 & 24.27 & 21.97 & 28.4 & 2.59 & 21.37 & 19.68 & 23.64 & 1.17 & 20.23 & 19.68 & 22.03 & 0.63 \\
\hline & 2013 & 22.57 & 21.36 & 23.6 & 0.66 & 22.53 & 20.02 & 23.81 & 0.97 & 20.49 & 19.49 & 22.08 & 1.13 \\
\hline \multirow[t]{3}{*}{2} & 2011 & 22.05 & 19.54 & 23.41 & 1.09 & 21.86 & 21.62 & 22.06 & 0.12 & 18.9 & 18.37 & 20.57 & 0.57 \\
\hline & 2012 & 18.7 & 18.07 & 20.57 & 0.69 & 22.02 & 21.76 & 22.58 & 0.23 & 23.97 & 19.14 & 26.95 & 1.87 \\
\hline & 2013 & 20.63 & 18.49 & 22.78 & 1.41 & 21.92 & 21.04 & 22.82 & 0.60 & 18.91 & 17.68 & 21.1 & 1.24 \\
\hline \multirow[t]{3}{*}{3} & 2011 & 18.82 & 17.99 & 20.31 & 0.52 & 21.96 & 21.28 & 22.49 & 0.35 & 22.55 & 20.75 & 23.99 & 0.93 \\
\hline & 2012 & 23.97 & 19.14 & 26.95 & 1.87 & 21.67 & 20.79 & 22.49 & 0.49 & 18.34 & 17.58 & 20.31 & 0.75 \\
\hline & 2013 & 18.65 & 17.47 & 20.58 & 1.24 & 21.92 & 20.41 & 23.77 & 1.05 & 21.54 & 19.31 & 23.07 & 1.02 \\
\hline \multirow[t]{3}{*}{4} & 2011 & 24.18 & 21.13 & 26.95 & 2.00 & 22.7 & 22.3 & 22.96 & 0.14 & 20.5 & 20.2 & 21.7 & 0.37 \\
\hline & 2012 & 26.68 & 22.57 & 28.31 & 1.44 & 22.8 & 22.3 & 23.19 & 0.21 & 20.4 & 20.04 & 21.7 & 0.42 \\
\hline & 2013 & 22.24 & 19.02 & 23.91 & 1.29 & 22.59 & 21.08 & 23.52 & 0.64 & 20.56 & 19.72 & 21.94 & 0.85 \\
\hline \multirow[t]{3}{*}{5} & 2011 & 21.53 & 20.95 & 22.33 & 0.33 & 21.35 & 20.87 & 22.5 & 0.41 & 19.86 & 18.42 & 21.83 & 0.96 \\
\hline & 2012 & 19.59 & 16.8 & 21.42 & 1.00 & 21.2 & 20.71 & 22.5 & 0.46 & 22.26 & 19.34 & 24 & 1.29 \\
\hline & 2013 & 21.75 & 20.33 & 22.9 & 0.80 & 21.56 & 20.38 & 22.96 & 0.91 & 19.94 & 19.09 & 20.89 & 0.49 \\
\hline \multirow[t]{3}{*}{6} & 2011 & 20.87 & 20.41 & 21.41 & 0.24 & 21.69 & 21.26 & 22.17 & 0.27 & 19.39 & 19.22 & 20.18 & 0.24 \\
\hline & 2012 & 22.13 & 20.8 & 23.26 & 0.77 & 21.41 & 20.19 & 22.17 & 0.50 & 19.49 & 19.18 & 20.19 & 0.33 \\
\hline & 2013 & 21.38 & 20.01 & 23.86 & 0.90 & 21.67 & 20.41 & 22.83 & 0.67 & 19.5 & 18.84 & 20.35 & 0.47 \\
\hline \multirow[t]{3}{*}{7} & 2011 & 22.35 & 21.84 & 22.96 & 0.35 & 22.39 & 22.01 & 22.9 & 0.26 & 19.3 & 18.9 & 20.39 & 0.36 \\
\hline & 2012 & 23.49 & 22.47 & 25.16 & 0.78 & 22.28 & 21.48 & 23.02 & 0.42 & 19.31 & 18.89 & 20.39 & 0.36 \\
\hline & 2013 & 22.21 & 20.31 & 23.65 & 1.15 & 22.32 & 19.93 & 23.19 & 0.83 & 19.32 & 18.43 & 20.35 & 0.58 \\
\hline \multirow[t]{3}{*}{8} & 2011 & 22.98 & 22.83 & 23.22 & 0.12 & 23.15 & 22.99 & 23.52 & 0.13 & 19.24 & 18.74 & 20.47 & 0.42 \\
\hline & 2012 & 24.38 & 22.91 & 27.09 & 1.78 & 23.14 & 22.76 & 23.52 & 0.17 & 19.18 & 18.72 & 20.47 & 0.45 \\
\hline & 2013 & 22.75 & 21.16 & 23.97 & 0.74 & 23.05 & 21.85 & 24 & 0.62 & 19.02 & 18.36 & 20.78 & 0.66 \\
\hline \multirow[t]{3}{*}{9} & 2011 & 18.57 & 18.22 & 19.19 & 0.27 & 21.75 & 21.21 & 23.2 & 0.53 & 21.72 & 21.28 & 21.97 & 0.16 \\
\hline & 2012 & 22.57 & 20.46 & 25.46 & 1.60 & 21.63 & 20.48 & 23.2 & 0.70 & 18.53 & 18.19 & 19.19 & 0.30 \\
\hline & 2013 & 18.69 & 17.95 & 19.74 & 0.52 & 21.61 & 18.26 & 23.49 & 1.30 & 21.88 & 20.72 & 22.91 & 0.62 \\
\hline \multirow[t]{3}{*}{10} & 2011 & 23.02 & 22.72 & 23.46 & 0.22 & 23.15 & 22.84 & 23.46 & 0.16 & 20.27 & 20.14 & 20.7 & 0.16 \\
\hline & 2012 & 24.77 & 22.83 & 28.24 & 2.39 & 23.33 & 22.84 & 23.78 & 0.29 & 20.23 & 20.04 & 20.7 & 0.18 \\
\hline & 2013 & 22.9 & 21.59 & 23.96 & 0.65 & 23.1 & 22.13 & 23.94 & 0.59 & 20.28 & 19.84 & 20.94 & 0.37 \\
\hline \multirow[t]{3}{*}{11} & 2011 & 22.47 & 22.28 & 22.84 & 0.17 & 23.29 & 22.43 & 23.7 & 0.29 & 19.14 & 18.77 & 19.92 & 0.28 \\
\hline & 2012 & 21.96 & 20.63 & 22.86 & 0.79 & 23.37 & 22.43 & 23.92 & 0.34 & 19.1 & 18.78 & 19.92 & 0.30 \\
\hline & 2013 & 22.57 & 21.56 & 23.59 & 0.57 & 23.29 & 22.35 & 23.97 & 0.46 & 19.21 & 18.5 & 20.52 & 0.59 \\
\hline \multirow[t]{3}{*}{12} & 2011 & 22.23 & 21.91 & 22.42 & 0.13 & 22.59 & 21.97 & 22.81 & 0.21 & 18.69 & 18.27 & 19.33 & 0.29 \\
\hline & 2012 & 18.56 & 18.05 & 19.33 & 0.39 & 22.56 & 21.97 & 23.35 & 0.34 & 22.99 & 21.91 & 25.61 & 1.14 \\
\hline & 2013 & 22.24 & 21.3 & 23.14 & 0.53 & 22.53 & 21.23 & 23.78 & 0.76 & 18.69 & 17.93 & 19.49 & 0.53 \\
\hline \multirow[t]{3}{*}{13} & 2011 & 21.47 & 21.02 & 21.9 & 0.25 & 21.28 & 20.86 & 21.84 & 0.26 & 18.6 & 18.23 & 18.87 & 0.17 \\
\hline & 2012 & 21.58 & 20.17 & 23.84 & 0.81 & 21.35 & 20.92 & 21.84 & 0.26 & 18.47 & 17.93 & 18.87 & 0.31 \\
\hline & 2013 & 21.36 & 19.7 & 23.14 & 1.03 & 21.44 & 20.07 & 22.58 & 0.78 & 18.57 & 17.62 & 19 & 0.43 \\
\hline \multirow[t]{3}{*}{14} & 2011 & 20.86 & 20.19 & 21.12 & 0.21 & 21.22 & 20.54 & 21.84 & 0.32 & 18.24 & 18.09 & 18.76 & 0.18 \\
\hline & 2012 & 20.97 & 20.19 & 22.48 & 0.55 & 20.7 & 19.96 & 22.11 & 0.45 & 18.21 & 18.02 & 18.76 & 0.19 \\
\hline & 2013 & 18.29 & 17.66 & 18.92 & 0.39 & 20.69 & 19.58 & 21.45 & 0.61 & 20.73 & 19 & 22.52 & 1.07 \\
\hline 15 & 2011 & 21.67 & 20.86 & 27.1 & 1.53 & 21.7 & 21.11 & 21.9 & 0.18 & 18.67 & 17.72 & 18.95 & 0.28 \\
\hline
\end{tabular}


Table 6 continued

\begin{tabular}{|c|c|c|c|c|c|c|c|c|c|c|c|c|c|}
\hline \multirow{2}{*}{$\begin{array}{l}\text { Location } \\
\text { Genotyp }\end{array}$} & \multirow[t]{2}{*}{ Year } & \multicolumn{4}{|l|}{ Dłon } & \multicolumn{4}{|c|}{ Poznań-Sołacz } & \multicolumn{4}{|c|}{ Złotniki } \\
\hline & & Mean & Min & Max & SD & Mean & Min & Max & SD & Mean & Min & $\operatorname{Max}$ & $\mathrm{SD}$ \\
\hline & 2012 & 26.27 & 22.27 & 27.61 & 1.35 & 21.81 & 21.11 & 22.58 & 0.34 & 18.57 & 17.72 & 18.96 & 0.30 \\
\hline & 2013 & 21.35 & 19.74 & 23.11 & 0.87 & 21.7 & 20.9 & 22.45 & 0.45 & 18.83 & 17.86 & 19.8 & 0.63 \\
\hline \multirow[t]{3}{*}{16} & 2011 & 20.51 & 20.07 & 20.85 & 0.18 & 21.41 & 21.21 & 21.85 & 0.16 & 17.69 & 16.97 & 17.91 & 0.24 \\
\hline & 2012 & 22.99 & 20.07 & 27.75 & 3.28 & 21.29 & 21.04 & 21.85 & 0.19 & 17.52 & 16.97 & 17.9 & 0.31 \\
\hline & 2013 & 20.83 & 18.81 & 22.79 & 1.09 & 21.27 & 20.13 & 22.57 & 0.73 & 17.52 & 16.81 & 18.51 & 0.68 \\
\hline \multirow[t]{3}{*}{17} & 2011 & 20.47 & 19.75 & 25.96 & 1.53 & 20.6 & 20.4 & 20.77 & 0.09 & 17.43 & 17.04 & 17.91 & 0.24 \\
\hline & 2012 & 24.8 & 16.62 & 28.23 & 2.64 & 20.64 & 20.29 & 21.19 & 0.24 & 17.29 & 16.79 & 17.91 & 0.34 \\
\hline & 2013 & 20.13 & 16.69 & 21.69 & 1.32 & 20.63 & 19.75 & 21.9 & 0.60 & 17.43 & 16.39 & 18.13 & 0.49 \\
\hline \multirow[t]{3}{*}{18} & 2011 & 20 & 19.49 & 20.55 & 0.28 & 20.31 & 20.14 & 20.73 & 0.17 & 19.29 & 18 & 19.99 & 0.93 \\
\hline & 2012 & 20.51 & 18.84 & 22.51 & 1.23 & 20.6 & 20.11 & 21.57 & 0.49 & 19.35 & 18 & 19.98 & 0.83 \\
\hline & 2013 & 20 & 18.54 & 21.55 & 0.96 & 20.33 & 19.67 & 21.48 & 0.53 & 19.36 & 18.03 & 19.95 & 0.73 \\
\hline \multirow[t]{3}{*}{19} & 2011 & 21.17 & 19.78 & 21.53 & 0.42 & 21.83 & 21.5 & 22.01 & 0.11 & 18.82 & 18.5 & 20.39 & 0.54 \\
\hline & 2012 & 21.98 & 19.78 & 23.9 & 1.27 & 21.84 & 21.21 & 22.27 & 0.25 & 18.81 & 18.39 & 19.96 & 0.51 \\
\hline & 2013 & 20.89 & 19.04 & 22.97 & 1.20 & 21.77 & 20.14 & 22.47 & 0.62 & 18.67 & 17.89 & 19.85 & 0.56 \\
\hline \multirow[t]{3}{*}{20} & 2011 & 21.28 & 20.77 & 21.42 & 0.19 & 21.98 & 21.71 & 22.12 & 0.10 & 17.96 & 17.75 & 18.33 & 0.15 \\
\hline & 2012 & 21.73 & 18.6 & 23.84 & 1.42 & 22.01 & 21.71 & 22.33 & 0.14 & 17.9 & 17.65 & 18.33 & 0.18 \\
\hline & 2013 & 21.42 & 19.43 & 22.71 & 1.01 & 21.94 & 21.41 & 22.56 & 0.45 & 17.92 & 17.47 & 18.51 & 0.29 \\
\hline \multirow[t]{3}{*}{21} & 2011 & 21.99 & 21.22 & 22.86 & 0.43 & 21.53 & 21.38 & 21.76 & 0.10 & 17.37 & 17.17 & 17.46 & 0.08 \\
\hline & 2012 & 22.46 & 20.89 & 23.99 & 1.03 & 21.56 & 21.29 & 22.04 & 0.17 & 17.31 & 17.13 & 17.45 & 0.10 \\
\hline & 2013 & 22.16 & 19.25 & 23.66 & 1.04 & 21.59 & 20.35 & 22.6 & 0.63 & 17.32 & 16.64 & 17.72 & 0.30 \\
\hline \multirow[t]{3}{*}{22} & 2011 & 21.96 & 21.79 & 22.29 & 0.12 & 21.87 & 21.38 & 22.28 & 0.26 & 19.54 & 18.02 & 19.83 & 0.43 \\
\hline & 2012 & 21.93 & 20.66 & 23.03 & 0.49 & 21.64 & 21 & 22.28 & 0.43 & 19.58 & 18.02 & 19.92 & 0.45 \\
\hline & 2013 & 22.11 & 21.17 & 23.17 & 0.55 & 21.62 & 20.42 & 22.6 & 0.76 & 19.47 & 18.03 & 19.97 & 0.57 \\
\hline \multirow[t]{3}{*}{23} & 2011 & 21.81 & 20.59 & 22.38 & 0.43 & 21.27 & 20.49 & 21.72 & 0.34 & 18.37 & 18.11 & 18.53 & 0.11 \\
\hline & 2012 & 22.55 & 20.59 & 25.68 & 1.55 & 20.9 & 19.91 & 21.72 & 0.64 & 18.24 & 17.84 & 18.53 & 0.24 \\
\hline & 2013 & 21.66 & 19.66 & 23.33 & 1.17 & 21.14 & 19.2 & 22.59 & 0.94 & 18.3 & 17.81 & 18.82 & 0.33 \\
\hline \multirow[t]{3}{*}{24} & 2011 & 21.43 & 20.94 & 22.33 & 0.32 & 21.37 & 20.86 & 22.45 & 0.41 & 17.26 & 16.81 & 17.94 & 0.33 \\
\hline & 2012 & 22.28 & 20.94 & 23.7 & 0.81 & 21.19 & 20.28 & 22.45 & 0.56 & 17.1 & 16.54 & 17.94 & 0.43 \\
\hline & 2013 & 21.33 & 19.58 & 23.27 & 1.13 & 21.22 & 19.79 & 22.61 & 0.71 & 17.37 & 16.35 & 18.13 & 0.57 \\
\hline \multirow[t]{3}{*}{25} & 2011 & 22.07 & 21.47 & 25.8 & 1.05 & 21.78 & 21.25 & 22.47 & 0.28 & 19.37 & 17.5 & 19.68 & 0.54 \\
\hline & 2012 & 25.26 & 21.57 & 26.91 & 1.47 & 21.54 & 20.8 & 22.47 & 0.46 & 19.44 & 19.04 & 19.68 & 0.18 \\
\hline & 2013 & 21.72 & 19.47 & 23.5 & 1.04 & 21.65 & 20.17 & 22.73 & 0.78 & 19.02 & 16.83 & 20 & 0.97 \\
\hline $\mathrm{LSD}_{0.05}$ & G: 0.1 & $Y: 0.07$ & 0.07 & $\mathrm{Y}: 0.3$ & SL: 0 . & 3, YL: 0 & 1, GYI & .57 & & & & & \\
\hline
\end{tabular}

Glucosinolates content was positive correlated with protein only in Sołacz (all 3 years) and negative correlated with ADF in Dłoń 2011 and Sołacz-all 3 years of study. Glucosinolates and oil content were negative correlate only in two environments-Sołacz 2013 and Złotniki 2012 (Table 10).
Particular traits have a different degree of significance and a different extent of contribution to the overall multi-trait variability. The multi-trait variability analysis includes identification of the most important traits in the objects' multi-trait variability. A statistical tool which helps solve this problem is 
Table 7 Protein content (\%) in twenty-five Brassica genotypes tested in nine environments

\begin{tabular}{|c|c|c|c|c|c|c|c|c|c|c|c|c|c|}
\hline \multirow{2}{*}{$\begin{array}{l}\text { Location } \\
\text { Genotyp }\end{array}$} & \multirow[t]{2}{*}{ Year } & \multicolumn{4}{|l|}{ Dłon } & \multicolumn{4}{|c|}{ Poznań-Sołacz } & \multicolumn{4}{|c|}{ Złotniki } \\
\hline & & Mean & Min & Max & SD & Mean & Min & Max & SD & Mean & Min & Max & SD \\
\hline \multirow[t]{3}{*}{1} & 2011 & 18.81 & 18.46 & 19.4 & 0.30 & 19.02 & 16.83 & 20.6 & 0.98 & 21.79 & 19.81 & 22.73 & 0.65 \\
\hline & 2012 & 19.73 & 18.4 & 21.25 & 1.08 & 19.83 & 16.83 & 21.87 & 1.50 & 22.28 & 19.81 & 23.02 & 0.86 \\
\hline & 2013 & 18.94 & 17.32 & 21.23 & 1.11 & 18.79 & 16.11 & 21.45 & 1.89 & 21.92 & 19.57 & 23.25 & 1.55 \\
\hline \multirow[t]{3}{*}{2} & 2011 & 20.73 & 18.93 & 23.55 & 1.59 & 20.67 & 19.12 & 21.54 & 0.56 & 22.30 & 19.67 & 23.46 & 0.92 \\
\hline & 2012 & 22.74 & 19.67 & 23.8 & 1.17 & 20.64 & 19.62 & 21.79 & 0.58 & 23.45 & 22.12 & 25.62 & 1.05 \\
\hline & 2013 & 18.98 & 16.54 & 21.71 & 1.67 & 20.59 & 18.03 & 22.00 & 1.06 & 22.34 & 19.06 & 23.88 & 1.99 \\
\hline \multirow[t]{3}{*}{3} & 2011 & 23.49 & 22.24 & 23.87 & 0.40 & 18.59 & 18.15 & 19.12 & 0.24 & 21.09 & 19.33 & 25.51 & 1.61 \\
\hline & 2012 & 23.45 & 22.12 & 25.62 & 1.05 & 18.88 & 18.3 & 19.59 & 0.37 & 23.80 & 23.10 & 25.63 & 0.69 \\
\hline & 2013 & 23.05 & 22.01 & 23.56 & 0.61 & 18.97 & 17.11 & 21.64 & 1.36 & 20.79 & 18.82 & 23.27 & 1.20 \\
\hline \multirow[t]{3}{*}{4} & 2011 & 20.33 & 19.19 & 22.03 & 1.01 & 18.46 & 18.33 & 18.57 & 0.07 & 22.90 & 21.21 & 23.29 & 0.52 \\
\hline & 2012 & 21.23 & 18.03 & 22.15 & 1.02 & 18.54 & 18.33 & 18.85 & 0.16 & 23.01 & 21.21 & 23.46 & 0.57 \\
\hline & 2013 & 18.51 & 16.2 & 22.09 & 1.55 & 18.51 & 17.21 & 19.99 & 0.77 & 22.73 & 20.94 & 23.98 & 1.15 \\
\hline \multirow[t]{3}{*}{5} & 2011 & 18.47 & 17.76 & 20.89 & 0.77 & 19.36 & 18.09 & 20.23 & 0.56 & 20.55 & 19.04 & 23.57 & 1.17 \\
\hline & 2012 & 20.40 & 19.01 & 23.57 & 1.18 & 19.73 & 18.09 & 20.70 & 0.77 & 21.41 & 20.36 & 22.99 & 0.76 \\
\hline & 2013 & 18.20 & 16.36 & 20.18 & 1.15 & 19.23 & 17.58 & 21.17 & 1.12 & 21.16 & 19.09 & 25.5 & 1.95 \\
\hline \multirow[t]{3}{*}{6} & 2011 & 19.85 & 19.32 & 21.72 & 0.56 & 20.07 & 19.52 & 20.37 & 0.20 & 23.33 & 22.49 & 23.52 & 0.25 \\
\hline & 2012 & 21.87 & 19.89 & 23.58 & 1.30 & 20.18 & 19.52 & 21.03 & 0.33 & 23.31 & 22.49 & 23.56 & 0.25 \\
\hline & 2013 & 20.23 & 17.8 & 22.75 & 1.30 & 20.04 & 18.83 & 22.24 & 0.91 & 23.16 & 22.37 & 23.85 & 0.53 \\
\hline \multirow[t]{3}{*}{7} & 2011 & 17.89 & 16.87 & 21.5 & 1.07 & 19.10 & 18.17 & 19.53 & 0.36 & 23.33 & 22.51 & 23.56 & 0.26 \\
\hline & 2012 & 22.64 & 16.12 & 23.63 & 1.89 & 19.10 & 18.17 & 19.88 & 0.45 & 23.34 & 22.51 & 23.55 & 0.27 \\
\hline & 2013 & 18.03 & 16.5 & 20.42 & 1.30 & 19.60 & 17.81 & 21.45 & 0.97 & 22.63 & 20.51 & 23.84 & 0.96 \\
\hline \multirow[t]{3}{*}{8} & 2011 & 17.81 & 17.37 & 18.24 & 0.25 & 18.84 & 18.07 & 19.21 & 0.29 & 23.19 & 22.71 & 23.3 & 0.14 \\
\hline & 2012 & 19.57 & 16.72 & 23.56 & 2.83 & 18.82 & 18.07 & 19.56 & 0.37 & 23.13 & 22.71 & 23.26 & 0.13 \\
\hline & 2013 & 17.69 & 15.19 & 19.12 & 1.01 & 18.71 & 17.37 & 20.64 & 1.02 & 23.08 & 22.49 & 23.72 & 0.37 \\
\hline \multirow[t]{3}{*}{9} & 2011 & 19.51 & 17.54 & 20.57 & 0.71 & 19.17 & 17.91 & 19.67 & 0.44 & 17.86 & 17.64 & 18.19 & 0.16 \\
\hline & 2012 & 18.70 & 17.52 & 23.08 & 1.39 & 18.81 & 17.91 & 19.68 & 0.57 & 19.87 & 19.41 & 23.55 & 1.05 \\
\hline & 2013 & 20.05 & 17.38 & 23.8 & 1.70 & 1 & 16.77 & 21.40 & 1.13 & 17.88 & 16.94 & 20.42 & 0.83 \\
\hline \multirow[t]{3}{*}{10} & 2011 & 18.60 & 17.76 & 19.39 & 0.45 & 19.30 & 18.55 & 20.11 & 0.36 & 23.38 & 23.33 & 23.47 & 0.03 \\
\hline & 2012 & 19.40 & 17.76 & 21.65 & 1.57 & 18.87 & 17.75 & 20.11 & 0.76 & 23.4 & 23.33 & 23.51 & 0.04 \\
\hline & 2013 & 18.35 & 17.29 & 21.21 & 1.11 & 19.12 & 17.58 & 21.59 & 1.14 & 23.4 & 23.08 & 23.54 & 0.12 \\
\hline \multirow[t]{3}{*}{11} & 2011 & 16.99 & 16.26 & 17.30 & 0.27 & 16.88 & 16.17 & 17.92 & 0.43 & 22.26 & 22.16 & 22.45 & 0.06 \\
\hline & 2012 & 18.06 & 15.87 & 20.20 & 1.52 & 16.65 & 15.73 & 17.92 & 0.55 & 22.27 & 22.16 & 22.45 & 0.07 \\
\hline & 2013 & 16.86 & 14.96 & 18.52 & 1.00 & 16.92 & 15.5 & 19.53 & 1.00 & 22.24 & 22.00 & 22.4 & 0.13 \\
\hline \multirow[t]{3}{*}{12} & 2011 & 18.19 & 17.75 & 18.45 & 0.21 & 18.84 & 18.42 & 20.21 & 0.42 & 23.23 & 23.15 & 23.41 & 0.07 \\
\hline & 2012 & 23.31 & 23.15 & 23.57 & 0.15 & 18.86 & 18.12 & 20.21 & 0.46 & 19.22 & 17.46 & 21.60 & 1.62 \\
\hline & 2013 & 17.98 & 16.54 & 19.57 & 0.85 & 18.79 & 17.02 & 21.84 & 1.28 & 23.27 & 22.87 & 23.68 & 0.25 \\
\hline \multirow[t]{3}{*}{13} & 2011 & 17.95 & 17.21 & 18.26 & 0.28 & 19.91 & 19.07 & 20.26 & 0.32 & 23.17 & 23.05 & 23.35 & 0.08 \\
\hline & 2012 & 18.12 & 17.02 & 19.39 & 0.62 & 19.76 & 19.07 & 20.25 & 0.37 & 23.24 & 23.05 & 23.53 & 0.16 \\
\hline & 2013 & 18.05 & 16.57 & 19.16 & 0.72 & 19.58 & 18.00 & 20.97 & 0.86 & 23.21 & 22.85 & 23.66 & 0.23 \\
\hline \multirow[t]{3}{*}{14} & 2011 & 18.29 & 17.76 & 20.17 & 0.61 & 19.95 & 19.07 & 20.42 & 0.35 & 23.52 & 23.37 & 23.66 & 0.09 \\
\hline & 2012 & 20.10 & 18.45 & 22.88 & 1.14 & 20.06 & 18.44 & 21.17 & 0.57 & 23.47 & 23.15 & 23.66 & 0.15 \\
\hline & 2013 & 23.50 & 23.15 & 23.84 & 0.22 & 20.26 & 19.23 & 21.77 & 0.63 & 18.38 & 15.66 & 20.45 & 1.25 \\
\hline 15 & 2011 & 18.79 & 17.82 & 20.35 & 0.66 & 19.55 & 19.14 & 20.52 & 0.33 & 23.11 & 22.90 & 23.71 & 0.20 \\
\hline
\end{tabular}


Table 7 continued

\begin{tabular}{|c|c|c|c|c|c|c|c|c|c|c|c|c|c|}
\hline \multirow{2}{*}{$\begin{array}{l}\text { Location } \\
\text { Genotyp }\end{array}$} & \multirow[t]{2}{*}{ Year } & \multicolumn{4}{|l|}{ Dłon } & \multicolumn{4}{|c|}{ Poznań-Sołacz } & \multicolumn{4}{|c|}{ Złotniki } \\
\hline & & Mean & Min & Max & SD & Mean & Min & $\operatorname{Max}$ & SD & Mean & Min & Max & $\mathrm{SD}$ \\
\hline & 2012 & 21.03 & 17.44 & 22.77 & 1.54 & 19.30 & 18.22 & 20.52 & 0.51 & 23.25 & 22.91 & 23.71 & 0.26 \\
\hline & 2013 & 18.26 & 16.20 & 19.56 & 1.06 & 19.56 & 18.20 & 20.79 & 0.81 & 23.25 & 22.34 & 23.91 & 0.57 \\
\hline \multirow[t]{3}{*}{16} & 2011 & 19.62 & 18.98 & 20.42 & 0.43 & 19.90 & 19.28 & 20.12 & 0.21 & 23.21 & 23.01 & 23.96 & 0.32 \\
\hline & 2012 & 19.63 & 18.22 & 20.75 & 0.86 & 19.99 & 19.28 & 20.27 & 0.24 & 23.30 & 23.03 & 23.99 & 0.30 \\
\hline & 2013 & 18.93 & 17.06 & 20.92 & 1.17 & 20.05 & 18.51 & 21.30 & 0.89 & 23.50 & 23.14 & 23.83 & 0.19 \\
\hline \multirow[t]{3}{*}{17} & 2011 & 19.74 & 18.53 & 22.57 & 0.91 & 20.39 & 20.15 & 20.51 & 0.10 & 23.45 & 23.01 & 23.99 & 0.38 \\
\hline & 2012 & 21.66 & 20.53 & 22.32 & 0.54 & 20.32 & 19.76 & 20.87 & 0.32 & 23.46 & 23.00 & 23.98 & 0.32 \\
\hline & 2013 & 19.29 & 17.92 & 21.64 & 1.27 & 20.36 & 19.27 & 21.90 & 0.70 & 23.62 & 23.05 & 23.87 & 0.25 \\
\hline \multirow[t]{3}{*}{18} & 2011 & 21.69 & 21.06 & 22.15 & 0.31 & 21.51 & 20.78 & 21.82 & 0.29 & 22.09 & 18.21 & 23.28 & 1.65 \\
\hline & 2012 & 22.09 & 19.76 & 23.6 & 1.13 & 21.03 & 19.96 & 21.89 & 0.69 & 22.31 & 18.12 & 23.55 & 1.73 \\
\hline & 2013 & 21.46 & 17.96 & 23.27 & 1.67 & 21.38 & 19.26 & 22.89 & 1.05 & 22.35 & 18.97 & 23.83 & 1.55 \\
\hline \multirow[t]{3}{*}{19} & 2011 & 19.17 & 18.61 & 21.16 & 0.61 & 19.29 & 18.79 & 19.92 & 0.29 & 21.32 & 18.90 & 21.97 & 1.13 \\
\hline & 2012 & 20.50 & 18.36 & 23.45 & 2.04 & 19.06 & 18.43 & 19.92 & 0.44 & 21.25 & 18.91 & 21.97 & 0.98 \\
\hline & 2013 & 19.41 & 16.82 & 22.06 & 1.97 & 19.41 & 17.99 & 21.79 & 1.03 & 21.12 & 19.06 & 21.97 & 0.87 \\
\hline \multirow[t]{3}{*}{20} & 2011 & 18.84 & 18.60 & 19.59 & 0.31 & 18.96 & 18.64 & 19.57 & 0.21 & 23.61 & 23.35 & 23.78 & 0.10 \\
\hline & 2012 & 20.39 & 18.43 & 23.00 & 1.90 & 18.95 & 18.69 & 19.57 & 0.22 & 23.64 & 23.35 & 23.86 & 0.13 \\
\hline & 2013 & 18.73 & 17.48 & 20.51 & 1.02 & 18.97 & 17.90 & 20.30 & 0.65 & 23.64 & 23.19 & 23.97 & 0.20 \\
\hline \multirow[t]{3}{*}{21} & 2011 & 18.28 & 17.06 & 19.49 & 0.60 & 19.75 & 19.61 & 19.96 & 0.10 & 23.12 & 23.01 & 23.23 & 0.05 \\
\hline & 2012 & 19.91 & 17.06 & 23.46 & 2.52 & 19.65 & 18.94 & 19.96 & 0.27 & 23.18 & 23.01 & 23.35 & 0.09 \\
\hline & 2013 & 18.01 & 16.45 & 22.71 & 1.50 & 19.69 & 18.58 & 21.58 & 0.87 & 23.39 & 23.04 & 23.99 & 0.35 \\
\hline \multirow[t]{3}{*}{22} & 2011 & 18.22 & 17.64 & 18.74 & 0.37 & 19.23 & 18.69 & 19.92 & 0.36 & 23.55 & 23.17 & 23.92 & 0.21 \\
\hline & 2012 & 20.03 & 17.26 & 23.8 & 2.67 & 19.56 & 18.69 & 20.48 & 0.63 & 23.44 & 23.08 & 23.89 & 0.27 \\
\hline & 2013 & 17.88 & 15.66 & 20.17 & 1.25 & 19.60 & 18.06 & 22.29 & 1.22 & 23.34 & 23.10 & 23.94 & 0.21 \\
\hline \multirow[t]{3}{*}{23} & 2011 & 18.63 & 18.35 & 18.91 & 0.16 & 20.11 & 19.52 & 20.93 & 0.38 & 23.29 & 22.97 & 23.84 & 0.25 \\
\hline & 2012 & 19.52 & 18.16 & 21.20 & 1.13 & 20.47 & 19.52 & 21.73 & 0.74 & 23.27 & 22.97 & 23.70 & 0.20 \\
\hline & 2013 & 18.57 & 17.02 & 20.78 & 1.04 & 20.13 & 18.46 & 22.34 & 1.04 & 23.06 & 22.82 & 23.46 & 0.17 \\
\hline \multirow[t]{3}{*}{24} & 2011 & 18.84 & 18.43 & 21.60 & 0.79 & 19.97 & 19.14 & 20.40 & 0.36 & 23.55 & 23.01 & 23.97 & 0.32 \\
\hline & 2012 & 21.76 & 17.34 & 22.89 & 1.34 & 20.14 & 19.14 & 20.99 & 0.49 & 23.58 & 23.09 & 23.99 & 0.29 \\
\hline & 2013 & 18.65 & 16.54 & 21.03 & 1.10 & 20.09 & 18.53 & 21.61 & 0.88 & 23.41 & 22.97 & 23.99 & 0.36 \\
\hline \multirow[t]{3}{*}{25} & 2011 & 17.67 & 17.11 & 20.48 & 0.80 & 19.13 & 18.10 & 19.93 & 0.43 & 23.58 & 23.04 & 23.91 & 0.19 \\
\hline & 2012 & 20.83 & 17.74 & 22.07 & 1.06 & 19.48 & 18.10 & 20.69 & 0.69 & 23.58 & 23.30 & 23.88 & 0.19 \\
\hline & 2013 & 17.69 & 16.10 & 20.24 & 0.98 & 19.14 & 17.93 & 20.69 & 0.98 & 23.24 & 22.24 & 23.85 & 0.44 \\
\hline $\mathrm{LSD}_{0.05}$ & G: 0.2 & Y: 0.0 & 0.08 & $Y: 0.3$ & 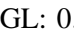 & YL: & GY & 65 & & & & & \\
\hline
\end{tabular}

canonical variable analysis. The results of the analysis for analyzed objects are presented in Table 11. The multidimensional analysis of the tested traits compared 25 genotypes in respect of five traits simultaneously in all nine environments data observations (Fig. 1). The first and second canonical varieties elucidated $87.98 \%$ and $5.81 \%$, respectively, of multivariate variability of genotypes (Fig. 1). Figure 1 shows the variability of analyzed traits in the configuration of the first two canonical variables. On the graph the coordinates of the point for particular genotypes are values of the first and second canonical variables, respectively. The most significant linear relation with the first canonical variable was observed for: NDF, ADF, oil content (positive correlation), protein and glucosinolates (negative correlations) 
Table 8 Oil content (\%) in twenty-five Brassica genotypes tested in nine environments

\begin{tabular}{|c|c|c|c|c|c|c|c|c|c|c|c|c|c|}
\hline \multirow{2}{*}{$\begin{array}{l}\text { Location } \\
\text { Genotyp }\end{array}$} & \multirow[t]{2}{*}{ Year } & \multicolumn{4}{|l|}{ Dłon } & \multicolumn{4}{|c|}{ Poznań-Sołacz } & \multicolumn{4}{|c|}{ Złotniki } \\
\hline & & Mean & Min & Max & SD & Mean & Min & Max & SD & Mean & Min & Max & SD \\
\hline \multirow[t]{3}{*}{1} & 2011 & 45.16 & 43.1 & 45.86 & 0.70 & 43.43 & 42.4 & 45.28 & 0.75 & 39.89 & 38.55 & 42.14 & 0.80 \\
\hline & 2012 & 43.85 & 40.68 & 45.48 & 1.59 & 42.86 & 41.56 & 45.28 & 0.96 & 39.48 & 37.8 & 43.84 & 1.66 \\
\hline & 2013 & 43.68 & 41.48 & 46.59 & 1.46 & 45.11 & 41.76 & 47.46 & 1.98 & 42.05 & 39.05 & 44 & 1.44 \\
\hline \multirow[t]{3}{*}{2} & 2011 & 42.84 & 38.82 & 46.18 & 2.41 & 43.33 & 43.02 & 43.76 & 0.23 & 41.28 & 39.98 & 43.49 & 0.89 \\
\hline & 2012 & 40.53 & 39.47 & 42.49 & 0.79 & 43.51 & 43.04 & 44.16 & 0.31 & 41.86 & 40.15 & 43.66 & 1.04 \\
\hline & 2013 & 43.38 & 41.68 & 45.72 & 1.52 & 43.45 & 41.02 & 45.07 & 1.05 & 42.37 & 40.84 & 43.49 & 0.78 \\
\hline \multirow[t]{3}{*}{3} & 2011 & 41.18 & 40 & 43.98 & 1.07 & 43.78 & 43.16 & 44.08 & 0.21 & 42.15 & 39.99 & 44.05 & 1.39 \\
\hline & 2012 & 41.86 & 40.15 & 43.66 & 1.04 & 43.91 & 43.16 & 44.74 & 0.46 & 40.66 & 36.03 & 44.77 & 2.10 \\
\hline & 2013 & 41.15 & 39.13 & 44.17 & 1.71 & 43.72 & 41.14 & 45.58 & 1.20 & 44.48 & 42.23 & 46.93 & 1.37 \\
\hline \multirow[t]{3}{*}{4} & 2011 & 43.17 & 41.26 & 44.72 & 1.10 & 43.04 & 42.5 & 44.69 & 0.61 & 41.14 & 38.51 & 43.94 & 1.60 \\
\hline & 2012 & 41.96 & 40.45 & 47.26 & 1.58 & 42.64 & 42.01 & 43.73 & 0.39 & 40.87 & 37.65 & 43.65 & 2.03 \\
\hline & 2013 & 43 & 39.27 & 47.76 & 2.72 & 42.85 & 40.97 & 45.19 & 1.34 & 40.35 & 39.11 & 42.4 & 0.98 \\
\hline \multirow[t]{3}{*}{5} & 2011 & 45.78 & 44.92 & 46.2 & 0.44 & 42.56 & 41.36 & 43.92 & 0.61 & 41.6 & 40.48 & 44.22 & 0.90 \\
\hline & 2012 & 41.47 & 38.29 & 45.57 & 1.96 & 41.86 & 40.03 & 43.92 & 1.18 & 44.14 & 40.1 & 45.81 & 1.84 \\
\hline & 2013 & 42.67 & 40.11 & 46.73 & 1.84 & 43.16 & 40.61 & 45.29 & 1.23 & 41.5 & 39.51 & 44.51 & 1.25 \\
\hline \multirow[t]{3}{*}{6} & 2011 & 43.83 & 41.02 & 44.98 & 1.53 & 41.74 & 41.02 & 42.94 & 0.86 & 39.08 & 38.56 & 40.19 & 0.54 \\
\hline & 2012 & 41.55 & 40.62 & 43.44 & 0.95 & 41.84 & 41.02 & 42.99 & 0.86 & 38.94 & 38.41 & 40.19 & 0.43 \\
\hline & 2013 & 43.73 & 41.29 & 47.22 & 2.18 & 42 & 41.15 & 43.17 & 0.60 & 38.42 & 37.28 & 39.95 & 0.83 \\
\hline \multirow[t]{3}{*}{7} & 2011 & 46.22 & 41.21 & 47.26 & 1.42 & 42.23 & 41.81 & 43.58 & 0.45 & 42.05 & 38.76 & 43.13 & 1.11 \\
\hline & 2012 & 41.27 & 39.96 & 48.29 & 2.05 & 42.36 & 41.56 & 44.18 & 0.72 & 42.5 & 40.88 & 44.04 & 0.65 \\
\hline & 2013 & 46.59 & 44.31 & 48.68 & 1.20 & 41.71 & 39.6 & 43.82 & 1.29 & 42.91 & 40.85 & 44.62 & 1.06 \\
\hline \multirow[t]{3}{*}{8} & 2011 & 45.47 & 44.43 & 46.05 & 0.39 & 43.85 & 41.44 & 44.99 & 1.29 & 42.57 & 36.7 & 45.9 & 2.21 \\
\hline & 2012 & 45.39 & 43.79 & 46.94 & 0.88 & 43.39 & 42 & 44.94 & 1.23 & 40.9 & 36.5 & 42.74 & 2.01 \\
\hline & 2013 & 46.06 & 44.94 & 48.69 & 0.98 & 43.12 & 39.14 & 44.94 & 1.48 & 41.07 & 36.99 & 42.17 & 1.35 \\
\hline \multirow[t]{3}{*}{9} & 2011 & 41.71 & 40.26 & 45.42 & 1.44 & 42.16 & 41.67 & 43.65 & 0.54 & 47.04 & 46.46 & 47.45 & 0.29 \\
\hline & 2012 & 46.48 & 44.37 & 48.48 & 1.05 & 42.44 & 41.14 & 43.66 & 0.84 & 40.33 & 36.8 & 43.12 & 1.92 \\
\hline & 2013 & 41.88 & 39.98 & 43.72 & 1.23 & 42.35 & 39.5 & 45.52 & 1.61 & 46.88 & 44.02 & 48.31 & 1.19 \\
\hline \multirow[t]{3}{*}{10} & 2011 & 43.8 & 42.82 & 44.7 & 0.50 & 40.55 & 39.06 & 41.58 & 0.58 & 42.28 & 40.29 & 46.75 & 2.04 \\
\hline & 2012 & 43.76 & 40.64 & 45.68 & 1.33 & 41.19 & 39.06 & 42.69 & 1.07 & 42.2 & 40.05 & 46.52 & 2.23 \\
\hline & 2013 & 44.39 & 39.84 & 47.39 & 1.83 & 41.4 & 37.59 & 43.49 & 1.55 & 41.36 & 35.62 & 46.05 & 2.89 \\
\hline \multirow[t]{3}{*}{11} & 2011 & 45.95 & 43.9 & 46.46 & 0.60 & 43.25 & 42.23 & 44.03 & 0.51 & 47.51 & 36.37 & 96.93 & 20.21 \\
\hline & 2012 & 44.92 & 41.75 & 47.88 & 1.63 & 43.75 & 42.23 & 45.65 & 0.90 & 40.08 & 36.42 & 45.54 & 2.92 \\
\hline & 2013 & 46.27 & 43.95 & 47.88 & 1.28 & 43.35 & 40.15 & 44.68 & 1.20 & 39.73 & 35.69 & 46.38 & 3.58 \\
\hline \multirow[t]{3}{*}{12} & 2011 & 43.06 & 42.77 & 43.44 & 0.18 & 42.78 & 41.35 & 43.5 & 0.49 & 42.5 & 41.99 & 43.01 & 0.30 \\
\hline & 2012 & 42.34 & 41.69 & 43.01 & 0.41 & 42.76 & 41.33 & 43.83 & 0.54 & 43.47 & 42.77 & 44.64 & 0.50 \\
\hline & 2013 & 43.71 & 42.02 & 45.77 & 0.97 & 42.65 & 39.36 & 45.45 & 1.43 & 42.5 & 41.5 & 43.18 & 0.58 \\
\hline \multirow[t]{3}{*}{13} & 2011 & 43.07 & 40.45 & 47.75 & 1.87 & 41.75 & 41.14 & 43.05 & 0.56 & 43.81 & 42.18 & 44.43 & 0.51 \\
\hline & 2012 & 41.71 & 39.76 & 43.95 & 1.56 & 41.79 & 41.23 & 43.05 & 0.56 & 43.79 & 42.18 & 44.43 & 0.53 \\
\hline & 2013 & 44.9 & 42.12 & 48.7 & 2.30 & 41.67 & 39.23 & 45.07 & 1.60 & 43.53 & 42.27 & 44.93 & 0.85 \\
\hline \multirow[t]{3}{*}{14} & 2011 & 41.01 & 40.08 & 44.22 & 1.20 & 41.65 & 40.41 & 43.05 & 0.66 & 39.21 & 39.04 & 39.71 & 0.17 \\
\hline & 2012 & 42.96 & 40.1 & 45.49 & 1.78 & 42.09 & 39.61 & 44.73 & 1.61 & 39.28 & 39 & 39.71 & 0.20 \\
\hline & 2013 & 39.34 & 38.28 & 40.12 & 0.62 & 42.7 & 41.47 & 44.27 & 0.92 & 43.64 & 40.6 & 50.45 & 3.23 \\
\hline 15 & 2011 & 42.8 & 41.78 & 43.56 & 0.55 & 42.58 & 41.65 & 42.98 & 0.31 & 44.57 & 42.35 & 45.77 & 1.23 \\
\hline
\end{tabular}


Table 8 continued

\begin{tabular}{|c|c|c|c|c|c|c|c|c|c|c|c|c|c|}
\hline \multirow{2}{*}{$\begin{array}{l}\text { Location } \\
\text { Genotyp }\end{array}$} & \multirow[t]{2}{*}{ Year } & \multicolumn{4}{|l|}{ Dłon } & \multicolumn{4}{|c|}{ Poznań-Sołacz } & \multicolumn{4}{|c|}{ Złotniki } \\
\hline & & Mean & Min & Max & SD & Mean & Min & Max & SD & Mean & Min & Max & $\mathrm{SD}$ \\
\hline & 2012 & 42.77 & 40.08 & 47.19 & 1.84 & 42.79 & 41.65 & 43.65 & 0.43 & 43.96 & 42.12 & 45.84 & 1.36 \\
\hline & 2013 & 42.44 & 40.04 & 47.16 & 1.78 & 42.56 & 40.63 & 44.75 & 1.01 & 43.35 & 42.17 & 46.19 & 1.25 \\
\hline \multirow[t]{3}{*}{16} & 2011 & 46.18 & 44.96 & 47.49 & 0.61 & 43.06 & 41.77 & 46.29 & 1.51 & 43.44 & 41.96 & 46.11 & 1.32 \\
\hline & 2012 & 46.18 & 42.8 & 48.97 & 1.53 & 43.35 & 41.43 & 46.14 & 1.72 & 42.84 & 41.94 & 45.48 & 1.02 \\
\hline & 2013 & 46.96 & 44.52 & 49.89 & 1.65 & 43.11 & 41.23 & 45.64 & 1.24 & 42.71 & 41.44 & 44.41 & 0.88 \\
\hline \multirow[t]{3}{*}{17} & 2011 & 45.02 & 42.43 & 46.17 & 0.94 & 41.59 & 41.16 & 42.42 & 0.33 & 40.91 & 40.16 & 42 & 0.51 \\
\hline & 2012 & 44.48 & 42.07 & 47.2 & 1.70 & 41.91 & 40.88 & 43.33 & 0.87 & 39.6 & 35.35 & 42 & 2.48 \\
\hline & 2013 & 45.6 & 40.44 & 48.38 & 2.14 & 41.52 & 38.9 & 43.4 & 1.30 & 41.16 & 35.91 & 45.64 & 1.99 \\
\hline \multirow[t]{3}{*}{18} & 2011 & 41.66 & 40.63 & 43.07 & 0.73 & 38.47 & 36.51 & 43.44 & 1.90 & 39.05 & 37.19 & 43.54 & 2.09 \\
\hline & 2012 & 41.92 & 40.39 & 46.35 & 1.75 & 39.72 & 36.72 & 42.54 & 2.05 & 38.26 & 36.73 & 41.44 & 1.36 \\
\hline & 2013 & 41.91 & 37.6 & 45.58 & 2.74 & 39.72 & 31.22 & 43.95 & 2.94 & 38.53 & 36.29 & 43.65 & 2.05 \\
\hline \multirow[t]{3}{*}{19} & 2011 & 44.64 & 43.15 & 45.58 & 0.63 & 42.68 & 42.18 & 43 & 0.24 & 43.04 & 39.74 & 44.9 & 1.94 \\
\hline & 2012 & 43.87 & 39.3 & 45.67 & 2.01 & 42.68 & 42.09 & 43.4 & 0.41 & 42.42 & 37 & 47.93 & 3.30 \\
\hline & 2013 & 44.12 & 41.15 & 47.64 & 2.26 & 42.03 & 36.51 & 44.49 & 2.12 & 41.8 & 37.05 & 47.57 & 3.68 \\
\hline \multirow[t]{3}{*}{20} & 2011 & 45.85 & 45.15 & 46.17 & 0.30 & 42.81 & 42.5 & 43.23 & 0.18 & 41.45 & 38 & 41.9 & 0.97 \\
\hline & 2012 & 45.28 & 43.11 & 46.97 & 1.20 & 42.88 & 42.5 & 43.23 & 0.23 & 40.73 & 36.95 & 41.93 & 1.81 \\
\hline & 2013 & 45.92 & 43.14 & 47.68 & 1.42 & 42.76 & 41.45 & 43.62 & 0.65 & 39.51 & 36.3 & 41.84 & 1.96 \\
\hline \multirow[t]{3}{*}{21} & 2011 & 46.25 & 45.12 & 47.71 & 0.57 & 41.96 & 41.07 & 42.55 & 0.41 & 40.26 & 36.02 & 41.99 & 2.32 \\
\hline & 2012 & 45.93 & 42.49 & 48.46 & 1.66 & 42.21 & 41.66 & 43.17 & 0.35 & 40.85 & 36.29 & 41.92 & 1.84 \\
\hline & 2013 & 46.63 & 41.63 & 49.69 & 1.78 & 42.16 & 39.86 & 44.78 & 1.45 & 39.56 & 36.17 & 45.36 & 2.79 \\
\hline \multirow[t]{3}{*}{22} & 2011 & 46.19 & 45.49 & 47.1 & 0.44 & 42.35 & 41.71 & 43.36 & 0.41 & 41.09 & 36.43 & 43.58 & 3.16 \\
\hline & 2012 & 45.87 & 40.49 & 47.1 & 1.69 & 42.04 & 40.64 & 43.36 & 0.76 & 38.81 & 35.99 & 43.58 & 3.33 \\
\hline & 2013 & 46.82 & 42.01 & 49.65 & 1.88 & 41.92 & 38.37 & 44.21 & 1.48 & 40.45 & 35.9 & 43.9 & 3.50 \\
\hline \multirow[t]{3}{*}{23} & 2011 & 45.14 & 44.58 & 45.87 & 0.30 & 40.63 & 39.63 & 41.37 & 0.43 & 40.12 & 36.1 & 42.83 & 2.83 \\
\hline & 2012 & 44.94 & 41.07 & 46.91 & 1.32 & 40.17 & 38.88 & 41.37 & 0.80 & 38.54 & 35.2 & 42.75 & 3.06 \\
\hline & 2013 & 45.49 & 42.99 & 48.96 & 1.61 & 40.44 & 38.08 & 43.05 & 1.53 & 39.48 & 35.56 & 42.99 & 2.21 \\
\hline \multirow[t]{3}{*}{24} & 2011 & 46.94 & 43.19 & 47.45 & 1.06 & 42.64 & 42.11 & 43.73 & 0.42 & 40.83 & 37.69 & 41.51 & 1.18 \\
\hline & 2012 & 45.16 & 41.2 & 49.18 & 2.19 & 42.49 & 41.6 & 43.73 & 0.56 & 41.01 & 38.22 & 41.69 & 0.82 \\
\hline & 2013 & 46.97 & 44.7 & 49.12 & 1.33 & 41.69 & 39.89 & 43.61 & 1.22 & 40.66 & 38.41 & 41.84 & 1.13 \\
\hline \multirow[t]{3}{*}{25} & 2011 & 46.77 & 41 & 48.18 & 1.65 & 42.41 & 41.66 & 43.52 & 0.44 & 39.97 & 39.06 & 43.52 & 1.54 \\
\hline & 2012 & 44.76 & 41 & 47.75 & 2.06 & 42.08 & 40.89 & 43.52 & 0.71 & 40.11 & 39.06 & 43.98 & 1.83 \\
\hline & 2013 & 46.85 & 44.82 & 49.66 & 1.53 & 41.81 & 40 & 43.96 & 1.29 & 40.94 & 39.07 & 43.74 & 1.78 \\
\hline $\mathrm{LSD}_{0.05}$ & G: 0.4 & $Y: 0.1$ & 0.17 & 0.8 & SL: & YL: & $29, \mathrm{GYL}$ & 1.44 & & & & & \\
\hline
\end{tabular}

(Table 11). The largest differences with regard to all five traits (measured in Mahalanobis distances) were recorded for genotypes 6 and 11 (the Mahalanobis distance between them was 7.62). The largest similarities were recorded for 15 and 25 (1.50) (Table 12).

Mahalanobis distances between all pairs of genotypes across nine environments were presented in Table 13. The smallest average value of Mahalanobis distance from nine environmental we observed for genotypes 16 and 24 (1.61). However, the largest for lines 6 and 11 (11.71). The most stable pair (the smallest range of Mahalanobis distance) was 16 and 24 (1.96). However, the least stable pair (range equal to 23.93) was 6 and 11 (Table 13).

For Mahalanobis distances values in particular environments we observed large range in Sołacz 2011 
Table 9 Glucosinolate content (umol/g) in twenty-five Brassica genotypes tested in nine environments

\begin{tabular}{|c|c|c|c|c|c|c|c|c|c|c|c|c|c|}
\hline \multirow{2}{*}{$\begin{array}{l}\text { Location } \\
\text { Genotyp }\end{array}$} & \multirow[t]{2}{*}{ Year } & \multicolumn{4}{|l|}{ Dłon } & \multicolumn{4}{|c|}{ Poznań-Sołacz } & \multicolumn{4}{|c|}{ Złotniki } \\
\hline & & Mean & Min & Max & SD & Mean & Min & Max & SD & Mean & Min & Max & SD \\
\hline \multirow[t]{3}{*}{1} & 2011 & 12.38 & 11.63 & 13.12 & 0.45 & 14.43 & 11.00 & 16.81 & 1.50 & 12.54 & 9.62 & 15.67 & 1.47 \\
\hline & 2012 & 13.78 & 11.65 & 16.74 & 1.75 & 14.61 & 11 & 16.68 & 1.61 & 12.07 & 9.51 & 14.33 & 1.55 \\
\hline & 2013 & 12.32 & 10.45 & 16.54 & 1.88 & 11.71 & 8.74 & 14.82 & 1.76 & 10.03 & 9.15 & 11.67 & 0.81 \\
\hline \multirow[t]{3}{*}{2} & 2011 & 35.55 & 29.3 & 45.49 & 4.80 & 30.86 & 27.82 & 32.55 & 1.27 & 31.31 & 28.89 & 32.92 & 1.24 \\
\hline & 2012 & 30.1 & 27.1 & 32.92 & 2.09 & 30.29 & 20.41 & 45.2 & 5.51 & 30.88 & 23.81 & 37.67 & 3.76 \\
\hline & 2013 & 35.6 & 11.84 & 45.31 & 9.72 & 28.62 & 13.2 & 36.91 & 5.48 & 30.16 & 25.96 & 45.4 & 4.67 \\
\hline \multirow[t]{3}{*}{3} & 2011 & 27.48 & 17.05 & 43.92 & 5.44 & 26.45 & 23.95 & 28.12 & 0.89 & 28.12 & 23.13 & 31.97 & 2.73 \\
\hline & 2012 & 30.88 & 23.81 & 37.67 & 3.76 & 26.02 & 23.33 & 28.47 & 1.46 & 28.94 & 17.05 & 41.52 & 5.16 \\
\hline & 2013 & 31.39 & 26.88 & 33.8 & 1.82 & 28.16 & 17.72 & 35.27 & 4.83 & 29.37 & 19.01 & 49.1 & 8.27 \\
\hline \multirow[t]{3}{*}{4} & 2011 & 13.79 & 11.28 & 16.38 & 1.39 & 13.82 & 12.96 & 15.75 & 0.64 & 11.72 & 9.14 & 13.31 & 1.01 \\
\hline & 2012 & 14.57 & 12.58 & 21.74 & 2.16 & 14.27 & 12.61 & 19.29 & 1.62 & 12.41 & 9.14 & 14.27 & 1.42 \\
\hline & 2013 & 15.35 & 8.75 & 27.77 & 4.37 & 14.34 & 11.07 & 26.17 & 3.70 & 12.11 & 8.64 & 18.9 & 2.71 \\
\hline \multirow[t]{3}{*}{5} & 2011 & 31.64 & 28.51 & 45.55 & 4.08 & 30.93 & 23.93 & 40.9 & 4.55 & 27.69 & 15.15 & 32.97 & 4.60 \\
\hline & 2012 & 28.45 & 15.15 & 32.79 & 4.66 & 32.28 & 20.5 & 53.81 & 10.31 & 31.44 & 14.15 & 42.31 & 8.66 \\
\hline & 2013 & 29.22 & 14.45 & 45.93 & 9.65 & 25.56 & 14.13 & 33.41 & 5.35 & 32.13 & 27.06 & 45.24 & 4.09 \\
\hline \multirow[t]{3}{*}{6} & 2011 & 60.87 & 53.99 & 74.36 & 5.48 & 55.74 & 53.8 & 57.37 & 1.09 & 45.62 & 41.35 & 51.77 & 3.91 \\
\hline & 2012 & 62.98 & 58.38 & 69.79 & 3.61 & 55.36 & 47.51 & 60.06 & 3.40 & 47.06 & 41.01 & 51.82 & 3.52 \\
\hline & 2013 & 57.72 & 27.44 & 75.56 & 12.88 & 51.31 & 26.8 & 59.87 & 8.12 & 46.92 & 41.22 & 53.88 & 4.05 \\
\hline \multirow[t]{3}{*}{7} & 2011 & 16.66 & 11.29 & 22.28 & 3.62 & 15.1 & 13.12 & 16.76 & 0.88 & 14.29 & 12.1 & 15.41 & 0.94 \\
\hline & 2012 & 15.88 & 12.5 & 17.42 & 1.50 & 15.29 & 13.12 & 18.43 & 1.30 & 13.45 & 11.03 & 15.52 & 1.59 \\
\hline & 2013 & 15.09 & 10.91 & 23.93 & 4.34 & 17.02 & 12.42 & 56.13 & 10.89 & 12.19 & 10.37 & 14.35 & 1.24 \\
\hline \multirow[t]{3}{*}{8} & 2011 & 13.84 & 12.28 & 14.84 & 0.67 & 13.77 & 12.04 & 15.76 & 1.48 & 13.79 & 10.26 & 14.69 & 1.09 \\
\hline & 2012 & 13.67 & 10.11 & 16.81 & 1.96 & 15.2 & 12.04 & 21.09 & 2.69 & 13.37 & 10.26 & 14.88 & 1.23 \\
\hline & 2013 & 14.19 & 9.21 & 27.13 & 4.68 & 13.74 & 11.98 & 15.97 & 1.16 & 12.34 & 9.07 & 14.47 & 1.46 \\
\hline \multirow[t]{3}{*}{9} & 2011 & 30.18 & 29.54 & 30.62 & 0.30 & 31.39 & 23.44 & 33.6 & 2.78 & 35.9 & 23.05 & 45.47 & 6.65 \\
\hline & 2012 & 35.12 & 23.05 & 45.69 & 6.04 & 31.37 & 23.44 & 45.43 & 4.62 & 29.73 & 28.66 & 30.52 & 0.58 \\
\hline & 2013 & 25.75 & 17.8 & 30.78 & 4.65 & 29.25 & 14.85 & 47.49 & 7.94 & 30.51 & 13.37 & 45.32 & 10.49 \\
\hline \multirow[t]{3}{*}{10} & 2011 & 11.72 & 10.02 & 14.12 & 1.08 & 13.54 & 11.55 & 16.25 & 1.42 & 9.91 & 8.69 & 10.69 & 0.54 \\
\hline & 2012 & 12.76 & 9.67 & 19.84 & 2.46 & 12.87 & 11.31 & 16.25 & 1.66 & 9.47 & 7.8 & 10.69 & 1.00 \\
\hline & 2013 & 12.86 & 8.57 & 22.86 & 3.94 & 14.51 & 10.57 & 31 & 5.18 & 9.78 & 6.7 & 12.59 & 1.65 \\
\hline \multirow[t]{3}{*}{11} & 2011 & 12.71 & 7.59 & 17.54 & 2.70 & 9.55 & 8.51 & 11.46 & 0.71 & 10.37 & 6.1 & 19.11 & 2.71 \\
\hline & 2012 & 11.26 & 6.21 & 16.16 & 2.99 & 8.86 & 6.83 & 11.35 & 1.11 & 10.08 & 9.1 & 12.88 & 0.88 \\
\hline & 2013 & 10.87 & 7.02 & 16.61 & 3.38 & 10.59 & 7.36 & 18.15 & 3.31 & 9.96 & 7.1 & 16.78 & 2.05 \\
\hline \multirow[t]{3}{*}{12} & 2011 & 17.25 & 13.13 & 23.42 & 2.35 & 16.53 & 14.29 & 18.55 & 1.27 & 17.85 & 10.56 & 31.53 & 6.54 \\
\hline & 2012 & 14.94 & 9.85 & 23.87 & 4.81 & 16.61 & 14.36 & 23.29 & 2.17 & 17.4 & 11.13 & 32.39 & 4.86 \\
\hline & 2013 & 14.81 & 6.7 & 32.44 & 6.43 & 15.56 & 10.41 & 23.98 & 4.25 & 15.13 & 10.2 & 23.22 & 4.62 \\
\hline \multirow[t]{3}{*}{13} & 2011 & 10.02 & 8.21 & 12.49 & 1.05 & 11.91 & 10.52 & 12.75 & 0.71 & 13.78 & 6.66 & 29.4 & 7.45 \\
\hline & 2012 & 11.11 & 6.23 & 16.78 & 2.82 & 11.15 & 8.5 & 12.95 & 1.32 & 11.57 & 6.66 & 26.86 & 5.89 \\
\hline & 2013 & 9.95 & 7.12 & 17.82 & 2.65 & 11.38 & 6.99 & 18.55 & 2.62 & 12.59 & 6.42 & 19.95 & 4.74 \\
\hline \multirow[t]{3}{*}{14} & 2011 & 38.62 & 33.12 & 42.63 & 2.66 & 32.99 & 30.52 & 37.02 & 1.55 & 33.85 & 27.64 & 45.89 & 4.48 \\
\hline & 2012 & 38.64 & 29.86 & 44.99 & 4.89 & 36.98 & 33.86 & 45.9 & 2.93 & 35.14 & 23.97 & 45.89 & 5.98 \\
\hline & 2013 & 36.27 & 31.26 & 41.96 & 4.00 & 36.63 & 30.76 & 45.46 & 3.63 & 34.38 & 19.85 & 45.94 & 7.55 \\
\hline 15 & 2011 & 30.75 & 19.67 & 33.1 & 3.36 & 21.79 & 18.68 & 27.9 & 2.00 & 22.76 & 15 & 23.91 & 2.17 \\
\hline
\end{tabular}


Table 9 continued

\begin{tabular}{|c|c|c|c|c|c|c|c|c|c|c|c|c|c|}
\hline \multirow{2}{*}{$\begin{array}{l}\text { Location } \\
\text { Genotyp }\end{array}$} & \multirow[t]{2}{*}{ Year } & \multicolumn{4}{|l|}{ Dłon } & \multicolumn{4}{|c|}{ Poznań-Sołacz } & \multicolumn{4}{|c|}{ Złotniki } \\
\hline & & Mean & Min & Max & SD & Mean & Min & Max & SD & Mean & Min & Max & SD \\
\hline & 2012 & 23.5 & 15.64 & 36.02 & 4.99 & 22.23 & 19.91 & 27.9 & 1.81 & 22.43 & 15 & 23.91 & 2.29 \\
\hline & 2013 & 28.16 & 12.04 & 42.46 & 7.25 & 22.53 & 13.32 & 32.75 & 5.34 & 20.29 & 15.02 & 23.99 & 4.05 \\
\hline \multirow[t]{3}{*}{16} & 2011 & 34.82 & 27.14 & 49.01 & 5.58 & 38.11 & 36.05 & 45.9 & 3.11 & 36.15 & 25.54 & 39.71 & 4.12 \\
\hline & 2012 & 32.53 & 27.03 & 45.9 & 5.79 & 37.15 & 20.96 & 45.3 & 5.00 & 37.28 & 32.04 & 40.83 & 2.59 \\
\hline & 2013 & 42.87 & 31.88 & 60.2 & 9.64 & 39.73 & 21.61 & 46.97 & 5.90 & 36.6 & 25.75 & 40.75 & 4.82 \\
\hline \multirow[t]{3}{*}{17} & 2011 & 23.64 & 20.3 & 28.51 & 2.17 & 27.7 & 26.53 & 32.98 & 1.53 & 30.26 & 22.27 & 51.67 & 9.76 \\
\hline & 2012 & 23.11 & 20.26 & 27.21 & 1.94 & 27.93 & 25.75 & 32.98 & 1.57 & 32.85 & 22.27 & 59.67 & 11.50 \\
\hline & 2013 & 21.83 & 11.03 & 48.45 & 9.02 & 29.54 & 21.54 & 45.9 & 7.23 & 27.73 & 20.96 & 61.76 & 12.57 \\
\hline \multirow[t]{3}{*}{18} & 2011 & 44.9 & 23.81 & 55.04 & 7.72 & 42.39 & 40.86 & 44.56 & 1.12 & 42.13 & 29.71 & 61.75 & 8.72 \\
\hline & 2012 & 43.33 & 18.35 & 55.5 & 10.89 & 42.44 & 40.32 & 46.69 & 1.78 & 37.12 & 30.89 & 55.15 & 6.07 \\
\hline & 2013 & 41.13 & 20.53 & 58.99 & 11.13 & 39.51 & 21.75 & 50.11 & 8.52 & 41.01 & 30.05 & 69.37 & 12.58 \\
\hline \multirow[t]{3}{*}{19} & 2011 & 37.04 & 21.32 & 44.77 & 7.80 & 32.81 & 27.58 & 45.93 & 5.36 & 25.96 & 23.78 & 29.78 & 1.41 \\
\hline & 2012 & 36.68 & 21.29 & 47.23 & 8.17 & 32.3 & 25.78 & 45.93 & 5.67 & 26.69 & 23.8 & 29.78 & 1.74 \\
\hline & 2013 & 30.08 & 16.2 & 51.23 & 10.67 & 32.53 & 19.5 & 45.17 & 7.35 & 28.97 & 22.19 & 42.6 & 4.82 \\
\hline \multirow[t]{3}{*}{20} & 2011 & 32.8 & 21.58 & 45.95 & 5.91 & 28.54 & 27.07 & 30.22 & 0.80 & 25.87 & 18.07 & 49.93 & 12.46 \\
\hline & 2012 & 33.46 & 21.58 & 48.8 & 7.38 & 29.1 & 26.37 & 33.03 & 1.67 & 28.16 & 18.14 & 49.5 & 12.45 \\
\hline & 2013 & 28.86 & 13.34 & 50.05 & 10.17 & 30.14 & 22.12 & 39.78 & 4.45 & 22.4 & 12.19 & 42.23 & 6.59 \\
\hline \multirow[t]{3}{*}{21} & 2011 & 38.88 & 22.84 & 45.78 & 6.27 & 30.52 & 29.24 & 31.74 & 0.71 & 24.82 & 23.01 & 31.26 & 1.94 \\
\hline & 2012 & 35.48 & 22.84 & 45.62 & 6.93 & 30.24 & 27.59 & 31.74 & 1.19 & 25.5 & 21.02 & 31.6 & 3.35 \\
\hline & 2013 & 32.97 & 11.14 & 47.83 & 11.10 & 30.2 & 17.4 & 43.59 & 6.42 & 25.16 & 17.31 & 33.28 & 4.53 \\
\hline \multirow[t]{3}{*}{22} & 2011 & 41.48 & 32.81 & 45.73 & 3.92 & 38.81 & 32.59 & 45.87 & 4.53 & 36.88 & 25.05 & 47.52 & 8.98 \\
\hline & 2012 & 39.24 & 23.38 & 50.72 & 6.42 & 39.79 & 32.59 & 45.72 & 4.86 & 36.19 & 25.83 & 47.72 & 8.39 \\
\hline & 2013 & 44.07 & 29.14 & 60.07 & 9.54 & 35.23 & 21 & 47.04 & 8.51 & 35.64 & 19.16 & 47.89 & 8.28 \\
\hline \multirow[t]{3}{*}{23} & 2011 & 49.6 & 39.98 & 53.37 & 3.15 & 40.76 & 39.03 & 42.9 & 1.03 & 43.36 & 29.18 & 47.99 & 7.29 \\
\hline & 2012 & 46.8 & 26.45 & 53.44 & 7.88 & 41.99 & 39.03 & 47.7 & 2.49 & 40.19 & 29.21 & 47.99 & 8.50 \\
\hline & 2013 & 48.38 & 28.66 & 60.36 & 8.41 & 40.66 & 30.48 & 49.97 & 4.80 & 35.57 & 23.98 & 47.87 & 8.66 \\
\hline \multirow[t]{3}{*}{24} & 2011 & 40.02 & 20.09 & 61.41 & 13.06 & 40.11 & 38.71 & 41.31 & 0.71 & 30.07 & 14.89 & 49.13 & 10.05 \\
\hline & 2012 & 41.02 & 20.09 & 61.69 & 15.97 & 40.76 & 38.3 & 48.68 & 2.64 & 30.63 & 15.2 & 49.1 & 8.37 \\
\hline & 2013 & 44.87 & 20.57 & 66.54 & 13.28 & 40.6 & 27.28 & 50.12 & 6.39 & 33.61 & 23.38 & 42.98 & 8.63 \\
\hline \multirow[t]{3}{*}{25} & 2011 & 23.86 & 11.32 & 29.05 & 5.12 & 25.18 & 19 & 29.12 & 3.75 & 22.73 & 19.64 & 29.64 & 3.05 \\
\hline & 2012 & 25.8 & 20.05 & 38.64 & 5.11 & 25.19 & 19.02 & 30.15 & 3.96 & 21.65 & 17.41 & 25.85 & 2.36 \\
\hline & 2013 & 23.66 & 10.84 & 47.85 & 9.14 & 28.14 & 22.4 & 37.8 & 4.99 & 20.88 & 16.63 & 28.45 & 3.18 \\
\hline $\mathrm{LSD}_{0.05}$ & G: 1.3 & Y: 0.4 & L: 0.4 & GY: 2.2 & GL: 2 & 3, YL: & $1, \mathrm{GYI}$ & 4.04 & & & & & \\
\hline
\end{tabular}

with the largest mean value (Fig. 2). Generally, in 2011 Mahalanobis distance values were larger than in 2012 and 2013. In 2013 these values were the smallest (Fig. 2).

\section{Discussion}

Understanding the genetic basis of seed quality traits and environmental factors that influence their expression is important for efficient rapeseed breeding (Jiang et al. 2014). To utilize genetic diversity present in interspecific cross-derived Brassica lines originated from Department of Genetics and Plant Breeding 
Table 10 Correlation coefficients between observed traits calculated on the basis of genotypes' means in environments independent

\begin{tabular}{|c|c|c|c|c|c|c|}
\hline Trait & Year & Location & NDF & $\mathrm{ADF}$ & Protein content & Oil content \\
\hline \multirow[t]{9}{*}{$\mathrm{ADF}$} & 2011 & Dłoń & $0.4028 *$ & & & \\
\hline & 2012 & Dłoń & -0.0759 & & & \\
\hline & 2013 & Dłoń & $0.7359 * * *$ & & & \\
\hline & 2011 & Sołacz & $0.5707 * *$ & & & \\
\hline & 2012 & Sołacz & $0.6278 * * *$ & & & \\
\hline & 2013 & Sołacz & $0.70 * * *$ & & & \\
\hline & 2011 & Złotniki & $0.4375^{*}$ & & & \\
\hline & 2012 & Złotniki & $0.4518 *$ & & & \\
\hline & 2013 & Złotniki & $0.6767 * * *$ & & & \\
\hline \multirow[t]{9}{*}{ Protein content } & 2011 & Dłoń & $-0.6268 * * *$ & $-0.5341 * *$ & & \\
\hline & 2012 & Dłoń & -0.2771 & -0.1391 & & \\
\hline & 2013 & Dłoń & $-0.7512 * * *$ & $-0.8506 * * *$ & & \\
\hline & 2011 & Sołacz & $-0.6183 * * *$ & $-0.7951 * * *$ & & \\
\hline & 2012 & Sołacz & $-0.6489 * * *$ & $-0.7947 * * *$ & & \\
\hline & 2013 & Sołacz & $-0.7626^{* * *}$ & $-0.8077 * * *$ & & \\
\hline & 2011 & Złotniki & -0.2746 & $-0.6374 * * *$ & & \\
\hline & 2012 & Złotniki & $-0.4056^{*}$ & $-0.3985^{*}$ & & \\
\hline & 2013 & Złotniki & $-0.4032 *$ & $-0.7148 * * *$ & & \\
\hline \multirow[t]{9}{*}{ Oil content } & 2011 & Dłoń & 0.3036 & $0.4116^{*}$ & $-0.5677 * *$ & \\
\hline & 2012 & Dłoń & 0.1388 & 0.2375 & $-0.5576^{* *}$ & \\
\hline & 2013 & Dłoń & $0.5407 * *$ & $0.6259 * * *$ & $-0.7439 * * *$ & \\
\hline & 2011 & Sołacz & $0.4589 *$ & $0.5116 * *$ & $-0.6226 * * *$ & \\
\hline & 2012 & Sołacz & $0.47 *$ & $0.4602 *$ & $-0.5402 * *$ & \\
\hline & 2013 & Sołacz & $0.6287 * * *$ & $0.4997 *$ & $-0.5375^{* *}$ & \\
\hline & 2011 & Złotniki & $0.4416^{*}$ & 0.2152 & $-0.458^{*}$ & \\
\hline & 2012 & Złotniki & 0.0848 & 0.2937 & -0.2472 & \\
\hline & 2013 & Złotniki & $0.406^{*}$ & $0.4714 *$ & $-0.6136^{* *}$ & \\
\hline \multirow[t]{9}{*}{ Glucosinolates content } & 2011 & Dłoń & $-0.619 * * *$ & $-0.4405^{*}$ & 0.2911 & -0.0374 \\
\hline & 2012 & Dłoń & $-0.4853^{*}$ & -0.2911 & 0.1902 & 0.0985 \\
\hline & 2013 & Dłoń & $-0.6921 * * *$ & -0.3609 & 0.3868 & -0.0414 \\
\hline & 2011 & Sołacz & $-0.6939 * * *$ & $-0.6386 * * *$ & $0.6139 * *$ & -0.3632 \\
\hline & 2012 & Sołacz & $-0.7339 * * *$ & $-0.6735 * * *$ & $0.6444 * * *$ & -0.3849 \\
\hline & 2013 & Sołacz & $-0.7236 * * *$ & $-0.7243 * * *$ & $0.6676^{* * *}$ & $-0.4085^{*}$ \\
\hline & 2011 & Złotniki & $-0.5313^{* *}$ & -0.1574 & -0.117 & -0.3563 \\
\hline & 2012 & Złotniki & $-0.6487 * * *$ & -0.198 & 0.1246 & $-0.4079^{*}$ \\
\hline & 2013 & Złotniki & $-0.5312 * *$ & -0.0947 & -0.1928 & -0.169 \\
\hline
\end{tabular}

$* P<0.05 ; * * P<0.01 ; * * * P<0.001$

collection of PULS and improve this genotypes itself, it was crucial to evaluate the extent of genetic diversity and to determine which of the tested factors i.e. genotype, year or location has the most significant effect on studied quality traits. Our results provide evidence for large phenotypic variability within the studied Brassica lines for oil, protein, glucosinolates and fiber (ADF and NDF) levels in seeds. The results of the present study are supported by the findings of Marjenovic-Jeromela et al. (2011) and Abideen et al. 
Table 11 Correlation coefficients between the first two principal components and the observed traits

\begin{tabular}{lcc}
\hline Trait & First canonical variable $\mathrm{V}_{1}$ & Second canonical variable $\mathrm{V}_{2}$ \\
\hline $\mathrm{NDF}$ & $0.8477 * * *$ & 0.2385 \\
$\mathrm{ADF}$ & $0.7038^{* * *}$ & $0.4038^{*}$ \\
Protein content & $-0.5182^{* *}$ & 0.3466 \\
Oil content & $0.4474 *$ & $-0.417 *$ \\
Glucosinolates content & $-0.9969 * * *$ & 0.0642 \\
Percentage of elucidated multivariate variability & 87.98 & 5.81 \\
\hline
\end{tabular}

$* P<0.05 ; * * * P<0.001$

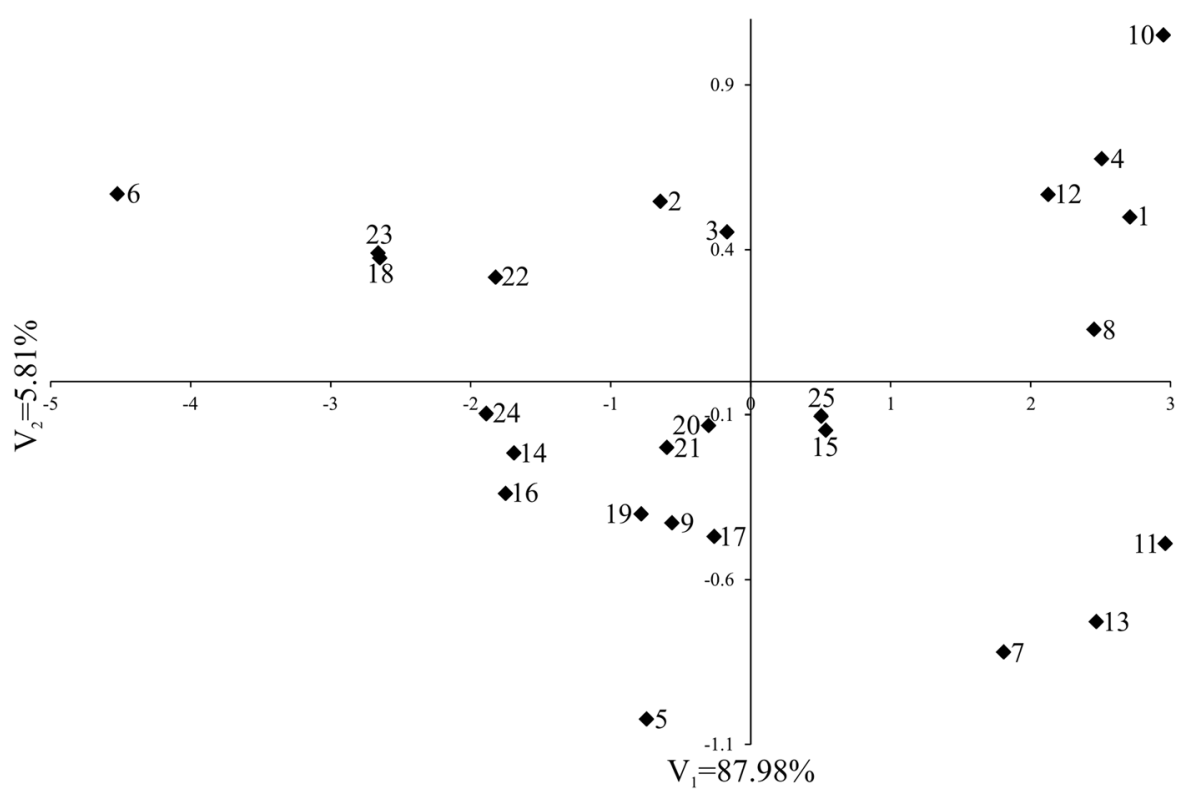

Fig. 1 Distribution of 25 Brassica genotypes in the space of two first canonical variables (Genotypes code see in Table 1)

(2013) who recorded significant variability in oil content and other quality traits in oilseed brassicas. Moreover, our results showed that all tested quality traits i.e. fiber, protein, oil and glucosinolate were significantly affected by environments. For example, a higher oil, fiber and glucosinolates content were observed in Dłoń than in Złotniki and Poznań-Sołacz. On the other hand protein content was in analyzed Brassica lines slightly higher in Złotniki than in other locations. Our results are in agreement with that of $\mathrm{Si}$ et al. (2003) who reported that environment had a much larger impact than genotype on protein content in canola seed, and its genetic variance and genotype $\times$ year $\times$ location interaction were significant. Similarly, Wu et al. (2005) claimed that since genotype $\times$ environment $(\mathrm{GE})$ interaction effects can cause genotype variation in seed quality traits across environments, the estimation of genetic main effect and GE interaction effect can demonstrate the degree of environmental influence on the genotypes and be useful for increasing selection efficiency for quality improvement.

In our study analysis of variance indicated that all interactions were also significant for all the traits of study (except year $\times$ location interaction for glucosinolates). Because of the fact that quality parameters are affected by genetic and environment, agronomic conditions, such as soil state, can affect seed quality parameters. In our experiment soil types and weather conditions differs among all locations. The results of 
Table 12 Mahalanobis distances between studied genotypes estimated on the basis of data form all environments

\begin{tabular}{|c|c|c|c|c|c|c|c|c|c|c|c|c|}
\hline & 1 & 2 & 3 & 4 & 5 & 6 & 7 & 8 & 9 & 10 & 11 & 12 \\
\hline 2 & 3.39 & & & & & & & & & & & \\
\hline 3 & 2.93 & 0.49 & & & & & & & & & & \\
\hline 4 & 0.81 & 3.23 & 2.77 & & & & & & & & & \\
\hline 5 & 3.85 & 1.78 & 1.78 & 3.68 & & & & & & & & \\
\hline 6 & 7.26 & 3.93 & 4.40 & 7.04 & 4.11 & & & & & & & \\
\hline 7 & 1.96 & 2.93 & 2.47 & 1.73 & 2.66 & 6.52 & & & & & & \\
\hline 8 & 0.55 & 3.14 & 2.66 & 0.83 & 3.48 & 7.01 & 1.49 & & & & & \\
\hline 9 & 3.50 & 1.62 & 1.66 & 3.58 & 1.49 & 4.29 & 3.07 & 3.25 & & & & \\
\hline 10 & 0.63 & 3.66 & 3.21 & 0.81 & 4.28 & 7.50 & 2.39 & 1.06 & 3.94 & & & \\
\hline 11 & 1.13 & 3.88 & 3.43 & 1.68 & 3.88 & 7.62 & 1.97 & 1.11 & 3.54 & 1.67 & & \\
\hline 12 & 0.77 & 2.91 & 2.48 & 1.32 & 3.51 & 6.75 & 2.15 & 0.93 & 2.94 & 1.18 & 1.43 & \\
\hline 13 & 1.32 & 3.42 & 2.95 & 1.66 & 3.36 & 7.16 & 1.34 & 1.08 & 3.24 & 1.90 & 0.97 & 1.47 \\
\hline 14 & 4.48 & 1.54 & 1.86 & 4.37 & 1.49 & 3.05 & 3.76 & 4.23 & 1.52 & 4.83 & 4.71 & 3.96 \\
\hline 15 & 2.33 & 1.39 & 0.95 & 2.20 & 1.68 & 5.13 & 1.59 & 1.96 & 1.74 & 2.72 & 2.64 & 1.99 \\
\hline 16 & 4.61 & 1.55 & 1.90 & 4.56 & 1.82 & 3.14 & 3.86 & 4.29 & 1.73 & 4.98 & 4.83 & 4.07 \\
\hline 17 & 3.18 & 1.16 & 1.00 & 3.11 & 1.21 & 4.47 & 2.30 & 2.85 & 1.42 & 3.59 & 3.39 & 2.74 \\
\hline 18 & 5.42 & 2.23 & 2.66 & 5.22 & 2.50 & 2.07 & 4.74 & 5.20 & 2.63 & 5.68 & 5.78 & 4.90 \\
\hline 19 & 3.61 & 1.13 & 1.23 & 3.56 & 1.00 & 3.92 & 2.89 & 3.30 & 0.76 & 4.01 & 3.76 & 3.10 \\
\hline 20 & 3.09 & 0.89 & 0.79 & 3.07 & 1.41 & 4.36 & 2.54 & 2.80 & 0.99 & 3.48 & 3.34 & 2.56 \\
\hline 21 & 3.39 & 0.91 & 0.97 & 3.36 & 1.28 & 4.09 & 2.79 & 3.10 & 0.87 & 3.78 & 3.61 & 2.85 \\
\hline 22 & 4.55 & 1.29 & 1.74 & 4.41 & 1.87 & 2.77 & 3.95 & 4.28 & 1.72 & 4.84 & 4.89 & 4.02 \\
\hline 23 & 5.38 & 2.14 & 2.60 & 5.25 & 2.51 & 2.00 & 4.80 & 5.15 & 2.36 & 5.66 & 5.70 & 4.81 \\
\hline 24 & 4.66 & 1.46 & 1.87 & 4.59 & 1.86 & 2.88 & 3.97 & 4.37 & 1.74 & 5.01 & 4.94 & 4.11 \\
\hline \multirow[t]{2}{*}{25} & 2.33 & 1.37 & 0.94 & 2.18 & 1.62 & 5.08 & 1.64 & 1.98 & 1.66 & 2.72 & 2.64 & 1.99 \\
\hline & 13 & 14 & 15 & 16 & 17 & 18 & 19 & 20 & 21 & 22 & 23 & 24 \\
\hline \multicolumn{13}{|l|}{2} \\
\hline \multicolumn{13}{|l|}{3} \\
\hline \multicolumn{13}{|l|}{4} \\
\hline \multicolumn{13}{|l|}{5} \\
\hline \multicolumn{13}{|l|}{6} \\
\hline \multicolumn{13}{|l|}{7} \\
\hline \multicolumn{13}{|l|}{8} \\
\hline \multicolumn{13}{|l|}{9} \\
\hline \multicolumn{13}{|l|}{10} \\
\hline \multicolumn{13}{|l|}{11} \\
\hline \multicolumn{13}{|l|}{12} \\
\hline \multicolumn{13}{|l|}{13} \\
\hline 14 & 4.21 & & & & & & & & & & & \\
\hline 15 & 2.12 & 2.37 & & & & & & & & & & \\
\hline 16 & 4.35 & 1.23 & 2.43 & & & & & & & & & \\
\hline 17 & 2.77 & 1.60 & 1.00 & 1.73 & & & & & & & & \\
\hline 18 & 5.27 & 1.20 & 3.36 & 1.88 & 2.64 & & & & & & & \\
\hline 19 & 3.31 & 1.08 & 1.47 & 1.27 & 0.90 & 2.21 & & & & & & \\
\hline 20 & 2.87 & 1.53 & 1.02 & 1.58 & 0.63 & 2.59 & 0.65 & & & & & \\
\hline
\end{tabular}


Table 12 continued

\begin{tabular}{lllllllllllll}
\hline & 13 & 14 & 15 & 16 & 17 & 18 & 19 & 20 & 21 & 22 & 23 \\
\hline 21 & 3.14 & 1.23 & 1.29 & 1.35 & 0.71 & 2.31 & 0.39 & 0.32 & & & \\
22 & 4.48 & 0.96 & 2.45 & 0.96 & 1.91 & 1.34 & 1.30 & 1.65 & 1.40 & \\
23 & 5.27 & 1.21 & 3.32 & 1.52 & 2.66 & 0.77 & 2.05 & 2.46 & 2.17 & 0.97 & \\
24 & 4.45 & 0.91 & 2.50 & 0.45 & 1.77 & 1.46 & 1.27 & 1.61 & 1.34 & 0.67 & 1.14 \\
25 & 2.16 & 2.31 & 0.19 & 2.44 & 1.02 & 3.29 & 1.42 & 1.00 & 1.26 & 2.40 & 3.26 & 2.49 \\
$\mathrm{D}_{0.05}=5.73$ & & & & & & & & & & \\
\hline
\end{tabular}

field trials conducted in three tested locations (Dłon, Złotniki, Poznań-Sołacz) confirmed the impact of soils and environmental conditions, especially water regime on particular seeds compound levels. Both, soils rich in fine clay particles, called 'heavy soils', which was available in Dłoń and luvisols, like those in Złotniki contains minerals and organic fractions sufficient to support cropping. This types of soils are used to be potentially very fertile when treated in the right way. On the other hand, in Poznań-Sołacz there are light, sandy soils. Moreover, the highest cumulative precipitation between three tested locations over a 3 year period were observed similarly like better soils in Dłoń and Złotniki. Thoroughly these conditions could be responsible for a higher oil, fiber and glucosinolates contents, observed in Dłoń than in Złotniki and Poznań-Sołacz.

It has been shown that canola protein content is affected by soil nitrogen level; higher nitrogen levels can lead to higher protein and glucosinolate contents and therefore lower oil contents (Aminpanah 2013). Similarly, high sulfur levels in soil, due to fertilizer, are known to increase the glucosinolate and the protein contents of canola seeds.

Furthermore, in canola, it is known that oil and protein contents follow an inverese relationship-the higher the oil and the lower the protein content. Seed protein content like other seed quality traits of rapeseed is a quantitative trait governed by additive gene action, so while many of the QTL for increased oil concentration are linked in coupling phase with QTL for decreased protein concentration (Gül et al. 2003), there are also many independent QTL for oil and protein concentration (Zhao et al. 2006). Therefore, in most cases, protein concentration will decrease when oil concentration is increased; however, there may also be cases where protein concentration is nearly maintained when oil concentration is increased. This literature data are confirmed by our results, since higher oil contents were observed in Dłoń compared to Złotniki, lower protein contents were expected in Dłoń when compared to Złotniki respectively. Besides, detailed analysis of these quality traits showed that in eight environments oil content was negative correlated with protein content and only in Złotniki 2012 these two traits were not correlated. Our results were consistent with those obtained by Khan et al. (2008) and Marjenovic-Jeromela et al. (2011), who reported significant and negative correlation between this two traits. However, this results were contrary to data obtained by Aytaç and Kinaci (2009), because they found significant and positive correlation between protein and oil content in Brassica oilseed.

Significant and positive association has been reported between protein content and glucosinolates (Ahmad et al. 2013). Our results exhibited that glucosinolates content was positive correlated with protein only in Sołacz (all 3 years). The differences between the results of the present study and those in previous studies could be due to the differences in the material and environmental conditions in which the studies were conducted.

According to Dimov et al. (2012) negative correlations between protein content of the meal and fibre content found in all Brassica DH populations is expected because selection for low fibre content will lead to an increase of protein content in the meal. Our results proved that protein content was exactly negative correlated almost in all analyzed environments with NDF (except Dłoń 2012 and Złotniki 2012) and ADF (except Dłoń 2012).

In the present study, it was found that particular traits have a different degree of significance and a different extent of contribution to overall multi-trait 
Table 13 Mahalanobis distances between genotypes across environments

\begin{tabular}{|c|c|c|c|c|c|c|c|c|}
\hline Comp. & Min-Max & Mean & Comp. & Min-Max & Mean & Comp. & Min-Max & Mean \\
\hline 1 versus 2 & $3.34-10.93$ & 6.26 & 3 versus 7 & $3.51-17.47$ & 7.67 & 5 versus 16 & $2.47-8.15$ & 4.93 \\
\hline 1 versus 3 & $3.25-11.46$ & 5.60 & 3 versus 8 & $3.10-13.25$ & 6.61 & 5 versus 17 & $1.55-8.60$ & 4.92 \\
\hline 1 versus 4 & $1.09-4.30$ & 2.54 & 3 versus 9 & $1.38-8.18$ & 4.43 & 5 versus 18 & $2.58-8.26$ & 4.90 \\
\hline 1 versus 5 & $3.09-9.95$ & 6.19 & 3 versus 10 & $3.82-13.21$ & 7.46 & 5 versus 19 & $1.54-5.95$ & 3.47 \\
\hline 1 versus 6 & $6.39-27.67$ & 10.98 & 3 versus 11 & $3.80-13.88$ & 7.30 & 5 versus 20 & $1.31-7.94$ & 4.41 \\
\hline 1 versus 7 & $2.15-17.64$ & 5.67 & 3 versus 12 & $2.63-12.43$ & 7.13 & 5 versus 21 & $1.19-8.38$ & 4.58 \\
\hline 1 versus 8 & $1.05-7.40$ & 3.33 & 3 versus 13 & $3.30-12.27$ & 6.55 & 5 versus 22 & $2.12-6.26$ & 4.40 \\
\hline 1 versus 9 & $4.18-9.49$ & 6.20 & 3 versus 14 & $1.51-13.01$ & 5.28 & 5 versus 23 & $3.18-7.53$ & 5.03 \\
\hline 1 versus 10 & $0.60-10.01$ & 3.50 & 3 versus 15 & $1.36-10.94$ & 4.94 & 5 versus 24 & $2.70-8.84$ & 5.20 \\
\hline 1 versus 11 & $1.86-6.68$ & 3.81 & 3 versus 16 & $2.07-11.03$ & 5.48 & 5 versus 25 & $1.41-6.66$ & 4.15 \\
\hline 1 versus 12 & $1.41-6.83$ & 4.07 & 3 versus 17 & $1.96-11.60$ & 5.03 & 6 versus 7 & $5.63-18.55$ & 8.81 \\
\hline 1 versus 13 & $2.36-5.27$ & 3.54 & 3 versus 18 & $2.17-10.60$ & 5.54 & 6 versus 8 & $5.58-25.68$ & 10.19 \\
\hline 1 versus 14 & $5.02-10.32$ & 6.84 & 3 versus 19 & $1.74-9.48$ & 4.63 & 6 versus 9 & $4.52-22.27$ & 9.11 \\
\hline 1 versus 15 & $2.84-6.13$ & 4.13 & 3 versus 20 & $0.79-10.64$ & 4.58 & 6 versus 10 & $6.45-28.29$ & 11.22 \\
\hline 1 versus 16 & $4.98-10.69$ & 6.94 & 3 versus 21 & $1.13-11.68$ & 5.40 & 6 versus 11 & $6.97-30.90$ & 11.71 \\
\hline 1 versus 17 & $3.45-8.97$ & 5.47 & 3 versus 22 & $1.86-11.91$ & 5.58 & 6 versus 12 & $6.72-26.82$ & 10.97 \\
\hline 1 versus 18 & $4.43-13.89$ & 7.67 & 3 versus 23 & $2.53-11.96$ & 6.29 & 6 versus 13 & $6.16-28.72$ & 10.87 \\
\hline 1 versus 19 & $3.15-8.85$ & 5.48 & 3 versus 24 & $2.29-11.97$ & 6.00 & 6 versus 14 & $2.93-23.37$ & 7.48 \\
\hline 1 versus 20 & $3.03-7.34$ & 5.19 & 3 versus 25 & $1.56-12.13$ & 4.71 & 6 versus 15 & $4.65-24.19$ & 8.53 \\
\hline 1 versus 21 & $3.34-8.88$ & 5.65 & 4 versus 5 & $2.38-8.88$ & 6.34 & 6 versus 16 & $3.17-21.06$ & 7.03 \\
\hline 1 versus 22 & $4.59-11.72$ & 6.54 & 4 versus 6 & $6.17-26.60$ & 10.51 & 6 versus 17 & $4.54-24.66$ & 8.64 \\
\hline 1 versus 23 & $4.92-12.32$ & 7.42 & 4 versus 7 & $2.35-16.06$ & 5.22 & 6 versus 18 & $1.74-18.74$ & 5.48 \\
\hline 1 versus 24 & $4.88-11.65$ & 7.26 & 4 versus 8 & $1.09-4.96$ & 3.16 & 6 versus 19 & $3.44-20.33$ & 7.14 \\
\hline 1 versus 25 & $3.05-5.87$ & 4.07 & 4 versus 9 & $2.89-9.59$ & 6.54 & 6 versus 20 & $4.02-21.15$ & 7.46 \\
\hline 2 versus 3 & $2.25-9.86$ & 6.31 & 4 versus 10 & $0.95-7.53$ & 2.68 & 6 versus 21 & $4.18-22.39$ & 7.94 \\
\hline 2 versus 4 & $3.34-10.27$ & 6.10 & 4 versus 11 & $1.83-6.30$ & 3.61 & 6 versus 22 & $1.96-18.52$ & 5.70 \\
\hline 2 versus 5 & $2.51-9.53$ & 4.67 & 4 versus 12 & $0.60-7.61$ & 3.65 & 6 versus 23 & $2.38-18.71$ & 5.49 \\
\hline 2 versus 6 & $3.52-20.87$ & 7.56 & 4 versus 13 & $1.77-5.78$ & 3.82 & 6 versus 24 & 2.84-19.79 & 6.89 \\
\hline 2 versus 7 & $2.93-16.46$ & 6.54 & 4 versus 14 & $4.47-9.77$ & 6.92 & 6 versus 25 & $4.27-24.20$ & 8.42 \\
\hline 2 versus 8 & $3.06-8.28$ & 5.58 & 4 versus 15 & $1.70-5.83$ & 3.77 & 7 versus 8 & $0.37-15.03$ & 3.97 \\
\hline 2 versus 9 & $2.65-9.99$ & 6.67 & 4 versus 16 & $4.56-11.21$ & 7.03 & 7 versus 9 & $4.43-18.00$ & 8.27 \\
\hline 2 versus 10 & $3.78-10.97$ & 6.74 & 4 versus 17 & $2.82-8.92$ & 5.79 & 7 versus 10 & $2.04-18.05$ & 5.51 \\
\hline 2 versus 11 & $3.84-15.84$ & 7.71 & 4 versus 18 & $4.15-13.92$ & 7.59 & 7 versus 11 & $1.49-20.62$ & 5.99 \\
\hline 2 versus 12 & $3.23-9.19$ & 5.71 & 4 versus 19 & $2.78-8.76$ & 5.57 & 7 versus 12 & $2.17-17.40$ & 6.60 \\
\hline 2 versus 13 & $3.31-12.10$ & 6.34 & 4 versus 20 & $2.84-7.16$ & 5.08 & 7 versus 13 & $1.60-17.60$ & 4.89 \\
\hline 2 versus 14 & $2.51-9.28$ & 5.67 & 4 versus 21 & $2.84-8.94$ & 5.79 & 7 versus 14 & $4.29-19.52$ & 7.67 \\
\hline 2 versus 15 & $1.58-8.89$ & 4.57 & 4 versus 22 & $3.71-11.44$ & 6.28 & 7 versus 15 & $1.61-15.98$ & 4.91 \\
\hline 2 versus 16 & $2.30-10.72$ & 4.86 & 4 versus 23 & $4.58-12.18$ & 7.21 & 7 versus 16 & $3.70-19.57$ & 7.19 \\
\hline 2 versus 17 & $2.27-10.95$ & 5.46 & 4 versus 24 & $4.57-11.96$ & 7.30 & 7 versus 17 & $2.37-18.70$ & 6.29 \\
\hline 2 versus 18 & $2.21-9.59$ & 4.78 & 4 versus 25 & $2.67-5.73$ & 4.06 & 7 versus 18 & $4.38-18.32$ & 7.53 \\
\hline 2 versus 19 & $1.21-9.09$ & 4.15 & 5 versus 6 & $4.01-22.07$ & 7.83 & 7 versus 19 & $2.47-16.69$ & 5.93 \\
\hline 2 versus 20 & $2.05-9.71$ & 4.69 & 5 versus 7 & $3.50-17.60$ & 7.08 & 7 versus 20 & $1.84-15.69$ & 5.37 \\
\hline 2 versus 21 & $2.28-10.94$ & 5.01 & 5 versus 8 & $3.01-11.49$ & 6.54 & 7 versus 21 & $2.23-17.59$ & 6.16 \\
\hline 2 versus 22 & $2.28-8.55$ & 4.79 & 5 versus 9 & $1.28-7.83$ & 4.86 & 7 versus 22 & $3.61-17.65$ & 6.47 \\
\hline 2 versus 23 & $1.87-9.54$ & 5.21 & 5 versus 10 & $3.71-14.04$ & 7.84 & 7 versus 23 & $4.15-18.17$ & 7.33 \\
\hline
\end{tabular}


Table 13 continued

\begin{tabular}{|c|c|c|c|c|c|c|c|c|}
\hline Comp. & Min-Max & Mean & Comp. & Min-Max & Mean & Comp. & Min-Max & Mean \\
\hline 2 versus 24 & $2.39-11.26$ & 5.19 & 5 versus 11 & $3.61-11.47$ & 7.01 & 7 versus 24 & $3.78-19.17$ & 7.18 \\
\hline 2 versus 25 & $2.00-9.08$ & 5.04 & 5 versus 12 & $2.38-9.19$ & 6.36 & 7 versus 25 & $1.41-17.55$ & 4.70 \\
\hline 3 versus 4 & $2.65-11.25$ & 6.00 & 5 versus 13 & $2.70-9.66$ & 6.19 & 8 versus 9 & $3.62-10.60$ & 7.09 \\
\hline 3 versus 5 & $0.76-12.26$ & 5.14 & 5 versus 14 & $1.99-7.55$ & 4.66 & 8 versus 10 & $1.28-5.82$ & 2.86 \\
\hline 3 versus 6 & $3.98-23.33$ & 8.95 & 5 versus 15 & $0.94-6.98$ & 4.44 & 8 versus 11 & $1.12-10.58$ & 3.94 \\
\hline 8 versus 12 & $1.10-8.52$ & 4.14 & 11 versus 17 & $2.90-11.40$ & 6.25 & 15 versus 21 & $1.32-4.43$ & 3.19 \\
\hline 8 versus 13 & $1.83-8.53$ & 3.61 & 11 versus 18 & $5.19-17.83$ & 8.76 & 15 versus 22 & $2.32-8.33$ & 4.28 \\
\hline 8 versus 14 & $4.28-11.39$ & 6.95 & 11 versus 19 & $3.24-12.37$ & 6.00 & 15 versus 23 & $3.10-8.72$ & 4.70 \\
\hline 8 versus 15 & $1.87-7.03$ & 3.65 & 11 versus 20 & $2.73-10.78$ & 5.44 & 15 versus 24 & $3.07-8.32$ & 4.61 \\
\hline 8 versus 16 & $4.19-12.41$ & 6.38 & 11 versus 21 & $2.95-11.34$ & 6.05 & 15 versus 25 & $1.24-4.38$ & 2.28 \\
\hline 8 versus 17 & $2.87-11.52$ & 5.48 & 11 versus 22 & $4.23-14.66$ & 7.03 & 16 versus 17 & $1.32-6.63$ & 2.86 \\
\hline 8 versus 18 & $4.64-14.83$ & 7.47 & 11 versus 23 & $4.82-15.61$ & 7.78 & 16 versus 18 & $2.54-7.24$ & 4.31 \\
\hline 8 versus 19 & $2.83-10.29$ & 5.14 & 11 versus 24 & $4.55-15.35$ & 7.67 & 16 versus 19 & $2.02-4.19$ & 3.11 \\
\hline 8 versus 20 & $2.26-9.27$ & 4.44 & 11 versus 25 & $1.97-8.70$ & 4.61 & 16 versus 20 & $2.01-5.30$ & 2.84 \\
\hline 8 versus 21 & $2.44-9.58$ & 4.83 & 12 versus 13 & $1.69-9.68$ & 4.26 & 16 versus 21 & $1.90-4.47$ & 2.77 \\
\hline 8 versus 22 & $3.80-12.63$ & 5.93 & 12 versus 14 & $4.47-10.47$ & 7.06 & 16 versus 22 & $1.48-4.51$ & 3.05 \\
\hline 8 versus 23 & $3.81-13.36$ & 6.77 & 12 versus 15 & $1.85-9.50$ & 4.67 & 16 versus 23 & $1.63-5.18$ & 3.01 \\
\hline 8 versus 24 & $4.12-13.01$ & 6.47 & 12 versus 16 & $4.46-11.38$ & 7.41 & 16 versus 24 & $0.78-2.74$ & 1.61 \\
\hline 8 versus 25 & $1.51-8.97$ & 3.53 & 12 versus 17 & $2.99-11.56$ & 6.37 & 16 versus 25 & $2.37-6.34$ & 4.01 \\
\hline 9 versus 10 & $3.97-12.61$ & 7.74 & 12 versus 18 & $4.38-13.17$ & 7.78 & 17 versus 18 & $2.20-8.81$ & 4.83 \\
\hline 9 versus 11 & $3.77-10.91$ & 6.95 & 12 versus 19 & $2.83-8.29$ & 5.67 & 17 versus 19 & $1.85-5.99$ & 3.54 \\
\hline 9 versus 12 & $2.95-10.77$ & 6.73 & 12 versus 20 & $2.75-10.68$ & 5.44 & 17 versus 20 & $1.73-5.61$ & 2.69 \\
\hline 9 versus 13 & $3.30-10.82$ & 6.68 & 12 versus 21 & $2.78-11.06$ & 5.94 & 17 versus 21 & $1.05-5.29$ & 2.56 \\
\hline 9 versus 14 & $2.20-11.39$ & 4.56 & 12 versus 22 & $3.73-10.92$ & 6.91 & 17 versus 22 & $1.87-8.22$ & 4.44 \\
\hline 9 versus 15 & $1.76-10.49$ & 5.15 & 12 versus 23 & $4.57-11.46$ & 7.49 & 17 versus 23 & $1.92-7.86$ & 4.20 \\
\hline 9 versus 16 & $2.43-12.11$ & 5.60 & 12 versus 24 & $4.47-11.81$ & 7.50 & 17 versus 24 & $0.50-7.38$ & 3.07 \\
\hline 9 versus 17 & $1.63-12.82$ & 5.43 & 12 versus 25 & $2.52-8.72$ & 5.11 & 17 versus 25 & $1.10-4.85$ & 2.98 \\
\hline 9 versus 18 & $3.00-9.09$ & 5.60 & 13 versus 14 & $4.07-9.76$ & 6.16 & 18 versus 19 & $2.11-7.34$ & 3.82 \\
\hline 9 versus 19 & $1.41-8.61$ & 3.75 & 13 versus 15 & $2.08-5.46$ & 3.21 & 18 versus 20 & $2.89-8.61$ & 4.64 \\
\hline 9 versus 20 & $0.92-11.93$ & 4.87 & 13 versus 16 & $4.33-12.58$ & 6.51 & 18 versus 21 & $2.55-7.65$ & 4.77 \\
\hline 9 versus 21 & $0.98-12.83$ & 5.16 & 13 versus 17 & $2.19-7.34$ & 4.69 & 18 versus 22 & $1.85-7.44$ & 3.66 \\
\hline 9 versus 22 & $1.52-10.17$ & 5.39 & 13 versus 18 & $4.59-14.22$ & 7.48 & 18 versus 23 & $1.50-6.71$ & 3.07 \\
\hline 9 versus 23 & $2.34-11.13$ & 5.68 & 13 versus 19 & $2.72-10.78$ & 5.37 & 18 versus 24 & $1.80-6.67$ & 4.21 \\
\hline 9 versus 24 & $2.17-13.26$ & 6.06 & 13 versus 20 & $2.60-9.36$ & 4.70 & 18 versus 25 & $2.75-9.74$ & 5.30 \\
\hline 9 versus 25 & $0.68-10.76$ & 5.03 & 13 versus 21 & $3.25-8.93$ & 5.18 & 19 versus 20 & $0.80-4.03$ & 2.10 \\
\hline 10 versus 11 & $1.93-11.49$ & 4.40 & 13 versus 22 & $4.17-13.61$ & 6.68 & 19 versus 21 & $1.04-4.26$ & 2.45 \\
\hline 10 versus 12 & $1.30-8.35$ & 4.41 & 13 versus 23 & $4.19-13.60$ & 7.07 & 19 versus 22 & $0.53-4.60$ & 2.91 \\
\hline 10 versus 13 & $2.38-9.94$ & 4.51 & 13 versus 24 & $4.21-13.42$ & 6.79 & 19 versus 23 & $1.78-4.53$ & 3.23 \\
\hline 10 versus 14 & $5.38-12.91$ & 8.02 & 13 versus 25 & $2.09-7.06$ & 4.01 & 19 versus 24 & $1.62-4.87$ & 3.08 \\
\hline 10 versus 15 & $2.92-9.75$ & 5.10 & 14 versus 15 & $2.68-5.78$ & 4.28 & 19 versus 25 & $1.08-4.34$ & 3.12 \\
\hline 10 versus 16 & $4.92-14.75$ & 8.00 & 14 versus 16 & $1.21-8.90$ & 3.95 & 20 versus 21 & $0.78-2.94$ & 1.68 \\
\hline 10 versus 17 & $3.49-13.42$ & 6.99 & 14 versus 17 & $1.53-8.79$ & 4.07 & 20 versus 22 & $1.04-4.66$ & 2.96 \\
\hline 10 versus 18 & $4.68-15.55$ & 8.36 & 14 versus 18 & $2.20-8.21$ & 4.42 & 20 versus 23 & $2.28-5.86$ & 3.32 \\
\hline 10 versus 19 & $3.28-12.88$ & 6.61 & 14 versus 19 & $2.01-6.49$ & 3.82 & 20 versus 24 & $1.92-5.60$ & 2.67 \\
\hline 10 versus 20 & $2.99-12.14$ & 6.07 & 14 versus 20 & $1.61-7.74$ & 4.00 & 20 versus 25 & $0.84-3.39$ & 2.36 \\
\hline
\end{tabular}


Table 13 continued

\begin{tabular}{|c|c|c|c|c|c|c|c|c|}
\hline Comp. & Min-Max & Mean & Comp. & Min-Max & Mean & Comp. & Min-Max & Mean \\
\hline 10 versus 21 & $3.16-11.55$ & 6.56 & 14 versus 21 & $1.90-7.84$ & 4.14 & 21 versus 22 & $1.11-5.62$ & 3.13 \\
\hline 10 versus 22 & $4.21-14.89$ & 7.15 & 14 versus 22 & $1.78-7.29$ & 4.10 & 21 versus 23 & $2.07-5.12$ & 3.17 \\
\hline 10 versus 23 & $4.85-14.82$ & 7.94 & 14 versus 23 & $1.21-6.64$ & 3.81 & 21 versus 24 & $1.14-4.69$ & 2.19 \\
\hline 10 versus 24 & $4.76-15.26$ & 8.12 & 14 versus 24 & $1.61-8.84$ & 4.16 & 21 versus 25 & $0.97-4.69$ & 2.67 \\
\hline 10 versus 25 & $2.88-11.14$ & 5.17 & 14 versus 25 & $2.34-8.17$ & 4.54 & 22 versus 23 & $1.04-3.01$ & 2.07 \\
\hline 11 versus 12 & $1.59-8.11$ & 4.37 & 15 versus 16 & $2.98-7.58$ & 4.26 & 22 versus 24 & $0.95-5.40$ & 2.67 \\
\hline 11 versus 13 & $1.41-8.39$ & 3.82 & 15 versus 17 & $1.79-5.24$ & 3.27 & 22 versus 25 & $1.35-7.00$ & 3.37 \\
\hline 11 versus 14 & $5.31-11.91$ & 7.31 & 15 versus 18 & $3.18-10.25$ & 5.47 & 23 versus 24 & $1.03-3.76$ & 2.49 \\
\hline 11 versus 15 & $3.13-9.16$ & 4.88 & 15 versus 19 & $1.95-5.45$ & 3.27 & 23 versus 25 & $2.24-7.56$ & 4.29 \\
\hline 11 versus 16 & $3.95-14.48$ & 7.51 & 15 versus 20 & $1.58-4.16$ & 2.83 & 24 versus 25 & $2.21-7.12$ & 4.11 \\
\hline
\end{tabular}

Fig. 2 Box-and-whisker diagram of the values of Mahalanobis distances, classified by the environments

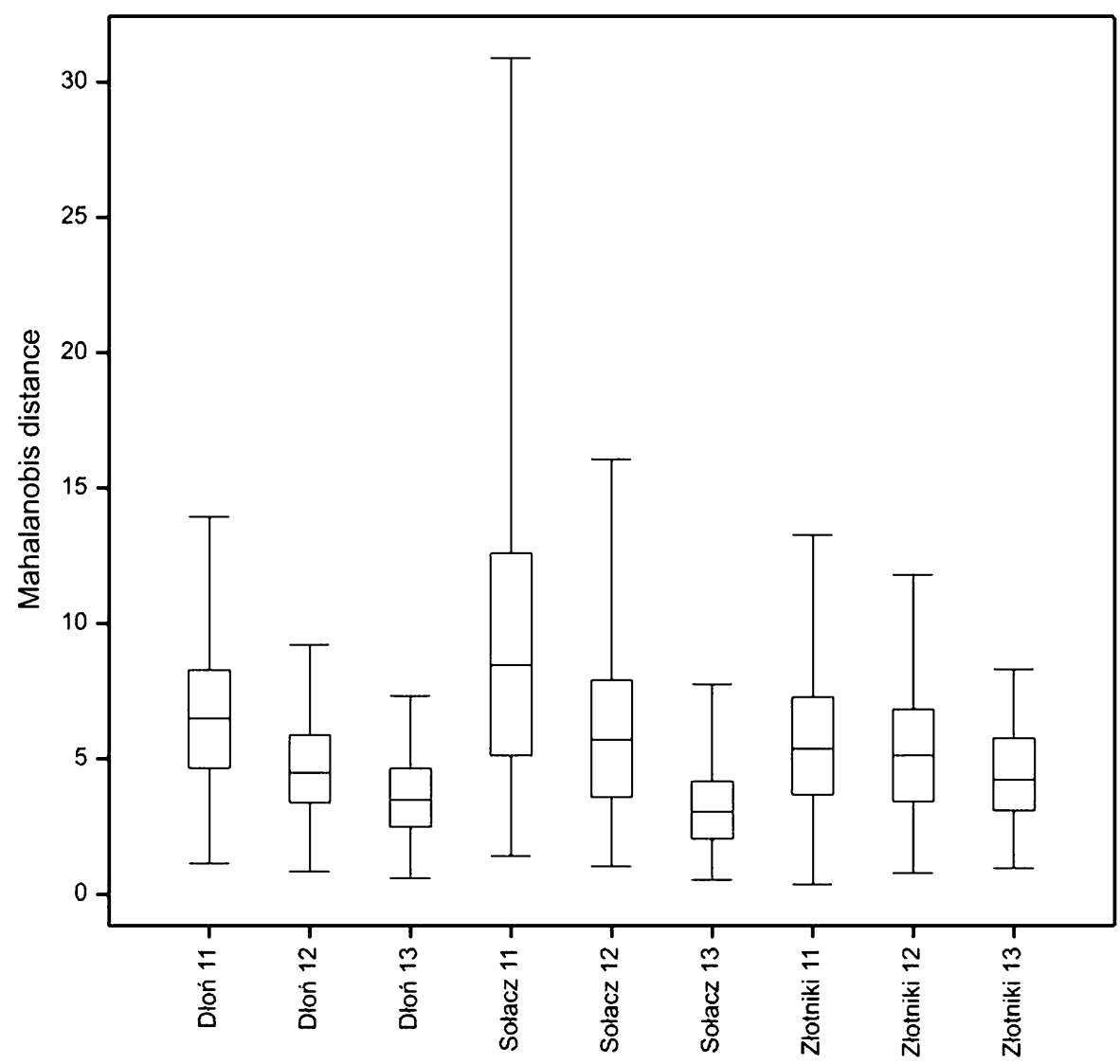

variability. The multidimentional analysis showed that the first and second canonical varieties elucidated $87.98 \%$ and $5.81 \%$ respectively of multivariate variability of genotypes. NDF, ADF and oil content showed a significant positive correlation with the first canonical variable. Quite opposite observations were made for protein and glucosinolate content, which were negative correlated. Our results are in contrary to 
Ahmad et al. (2013), who reported that all tested quality traits were positively correlated with oil content and with one another at both genotypic and phenotypic levels.

In our experiment the largest differences with regard to five tested traits occured between lines 6 and 11, which exhibited different origin (B.napus $\times B$. rapa ssp. pekinensis and B.napus $\times$ B. rapa ssp. trilocularis $c v$. Yellow Sarson respectively. However, the largest similarities were recorded for lines 15 and 25 (B.napus MS8 line $\times$ B. rapa ssp. pekinensis and B. napus line MS8 respectively).

Moreover, Brassica lines numbered as 14, 16, 21 and 22 showed superiority for oil content, whereas genotype 11 exhibited superiority for minimum glucosinolate content. These genotypes may further be utilized in breeding programmes for evolving rapeseed varieties having high oil content and with superior seeds quality.

Taking into account the effect of genotype and environment on all tested seed quality traits in interspecific cross-derived Brassica lines, it was demonstrated that genotype had significant impact on glucosinolate content while oil, protein and fiber contents were influenced mostly by environment.

As an extension of our research hereafter genomewide association studies should be used for the investigation of quantitative trait loci (QTL) linked to seed quality traits in winter oilseed rape (Brassica napus L.) genotypes. Genome-wide association analyses would enable identification of highly promising candidate genes and markers for breeding towards improved quality traits in rapeseed.

Acknowledgements The autors are grateful to Dr Grzegorz Budzianowski from the Plant Breeding Strzelce, Małyszyn Branch in Poland for the seed quality analysis. This study was funded by the Polish Ministry of Agriculture and Rural Development.

\section{Compliance with ethical standards}

Conflict of interest The authors declare that they have no conflict of interest.

Open Access This article is distributed under the terms of the Creative Commons Attribution 4.0 International License (http:// creativecommons.org/licenses/by/4.0/), which permits unrestricted use, distribution, and reproduction in any medium, provided you give appropriate credit to the original author(s) and the source, provide a link to the Creative Commons license, and indicate if changes were made.

\section{References}

Abbadi A, Leckband G (2011) Rapeseed breeding for oil content, quality, and sustainability. Eur J Lipid Sci Technol 113(10):1198-1206

Abideen SN, Nadeem F, Abideen SA (2013) Genetic variability and correlation studies in Brassica napus L. genotypes. IJIAS 2:574-581

Ahmad B, Mohammad S, Farooq-i-Azam Ali I, Ali J, Rehman S (2013) Studies of genetic variability, heritability and phenotypic correlations of some qualitative traits in advance mutantlines of winter rapeseed (Brassica napus L.). Am Eurasian J Agric Environ Sci 13:531-538

Aminpanah H (2013) Effect of nitrogen rate on seed yield, protein and oil content of two canola (Brassica napus L.) cultivars. Acta Agric Slov 101(2):183-190

Aytaç Z, Kinaci G (2009) Genetic variability and association studies of some quantitative characters in winter rapeseed (Brassica napus L.). Afr J Biotechnol 8:3547-3554

Bell JM (1984) Nutrients and toxicants in rapeseed meal: a review. J Anim Sci 58(4):996-1010

Dimov Z, Suprianto E, Hermann F, Möllers C (2012) Genetic variation for seed hull and fibre content in a collection of European winter oilseed rape material (Brassica napus L.) and development of NIRS calibrations. Plant Breed 131:361-368

Feng J, Long Y, Shi L, Shi JQ, Barker G, Meng JL (2012) Characterization of metabolite quantitative trait loci and metabolic networks that control glucosinolate concentration in the seeds and leaves of Brassica napus. New Phytol 193(1):96-108

Friedt W, Snowdon RJ (2010) Oilseed rape. In: Vollmann J, Istvan R (eds) Handbook of plant breeding, chapter vol. 4: oil crops breeding. Springer, Berlin, pp 91-126

Gül M, Becker HC, Ecke W (2003) QTL mapping and analysis of QTL $\times$ nitrogen interactions for protein and oil contents in Brassica napus L. In: Proceedings of the 11th international rapeseed congress, Copenhagen, Denmark, pp 91-93

Halkier BA, Gershenzon J (2006) Biology and biochemistry of glucosinolates. Annu Rev Plant Biol 57:303-333

Huang XQ, Huang T, Hou GZ, Li L, Hou Y, Lu YH (2016) Identification of QTLs for seed quality traits in rapeseed (Brassica napus L.) using recombinant inbred lines (RILs). Euphytica 210:1

Jiang CC, Shi JQ, Li RY, Long Y, Wang H, Li DR, Zhao J, Meng J (2014) Quantitative trait loci that control the oil content variation of rapeseed (Brassica napus L.). Theor Appl Genet 127(4):957-968

Khan HH, Rahman H, Ali AH (2008) Magnitude of heterosis and heritability in sunflower over environments. Pak J Bot $1: 301-308$

Körber N, Bus A, Li J, Parkin IAP, Wittkop B, Snowdon RJ, Stich B (2016) Agronomic and seed quality traits dissected by genome-wide association mapping in Brassica napus. Front Plant Sci 7:386

Liu Z, Hirani AH, McVetty PBE, Daayf F, Quiros CF, Li GY (2012) Reducing progoitrin and enriching glucoraphanin in Brassica napus seeds through silencing of the GSL-ALK gene family. Plant Mol Biol 79(1-2):179-189 
Liu G, Zhao Y, Gowda M, Longin CFH, Reif JC, Mette MF (2016) Predicting hybrid performances for quality traits through genomic-assisted approaches in central European Wheat. PLoS ONE 11(7):e0158635

Mahalanobis PC (1936) On the generalized distance in statistics. In: Proceedings on the National Institute of Science of India, vol 12, pp 49-55

Marjanovic-Jeromela A, Marinkovic R, Ivanovska S, Jankulovska $M$ et al (2011) Variability of yield determining components in winter rapeseed (Brassica napus L.) and their correlation with seed yield. Genetika 43:51-66

Möllers C (2004) Potential and future prospects for rapeseed oil. In: Gunstone FD (ed) Rapeseed and canola oil-production, processing, properties and uses. Blackwell Publishing, Oxford

Niemann J, Wojciechowski A, Janowicz J (2012) Broadening the variability of quality traits in rapeseed through interspecific hybridization with an application of immature embryo culture. BioTechnologia 93:109-115

Payne R, Murrey D, Harding S, Baird D, Soutou D (2012) Introduction to GenStat for Windows, 15th edn. VSN International, Hemel Hempstead

Rani T, Yadav RC, Yadav NR, Rani A, Singh D (2013) Genetic transformation in oilseed brassicas-a review. Indian $\mathrm{J}$ Agric Sci 83(4):367-373

Ratajczak K, Sulewska H, Szymańska G (2017) New winter oilseed rape varieties-seed quality and morphological traits depending on sowing date and rate. Plant Prod Sci 20(3):262-272

Rencher AC (1992) Interpretation of canonical discriminant functions, canonical variates, and principal components. Am Stat 46:217-225

Seidler-Łożykowska K, Bocianowski J (2012) Evaluation of variability of morphological traits of selected caraway (Carum carvi L.) genotypes. Ind Crops Prod 35:140-145

Shapiro SS, Wilk MB (1965) An analysis of variance test for normality (complete samples). Biometrika 52:591

Si P, Mailer RJ, Galwey N, Turner DW (2003) Influence of genotype and environment on oil and protein concentrations of canola (Brassica napus L.) grown across southern Australia. Crop Pasture Sci 54:397-407

Tanksley SD, Nelson JC (1996) Advanced backross QTL analysis: a method for the simultaneous discovery and transfer of valuable QTLs from unadapted germplasm into elite breeding lines. Theor Appl Genet 92(2):191-203

Vageeshbabu HS, Chopra VL (1997) Genetic and biotechnological approaches for reducing glucosinolates from rapeseed-mustard meal. J Plant Biochem Biotechnol $6(2): 53-62$

Velasco L, Becker H (1998) Estimating the fatty acid composition of the oil in intact-seed rapeseed (Brassica napus L.) by near-infrared reflectance spectroscopy. Euphytica 101(2):221-230

Velasco L, Mollers C, Becker HC (1999) Estimation of seed weight, oil content and fatty acid composition in intact single seeds of rapeseed (Brassica napus L.) by near-infrared reflectance spectroscopy. Euphytica 106:79-85

Wu JG, Shi CH, Zhang HZ (2005) Genetic analysis of embryo, cytoplasmic and maternal effects and their environment interactions for protein content in Brassica napus L. Aust J Agric Res 56:69-73

Würschum T, Liu W, Maurer HP, Abel S, Reif JC (2012) Dissecting the genetic architecture of agronomic traits in multiple segregating populations in rapeseed (Brassica napus L.). Theor Appl Genet 124:153-161

Zhao J, Becker HC, Zhang D, Zhang Y, Ecke W (2006) Conditional QTL mapping of oil content in rapeseed with respect to protein content and traits related to plant development and grain yield. Theor Appl Genet 113:33-38

Zhao J, Dimov Z, Becker HC, Ecke W, Möllers C (2007) Mapping QTL controlling fatty acid composition in a doubled haploid rapeseed population segregating for oil content. Mol Breed 21:115-125

Zou J, Zhao Y, Liu P, Shi L, Wang X et al (2016) Seed quality traits can be predicted with high accuracy in Brassica napus using genomic data. PLoS ONE 11(11):e016662 